\title{
MiRNA-296-3p-ICAM-1 axis promotes metastasis of prostate cancer by possible enhancing survival of natural killer cell-resistant circulating tumour cells
}

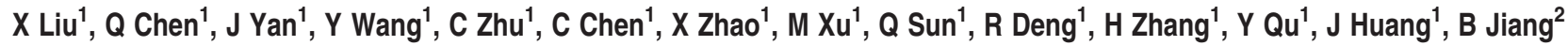 \\ and $\mathrm{J} \mathrm{Yu}^{*, 1,2,3}$
}

Natural killer (NK) cells are important in host to eliminate circulating tumour cells (CTCs) in turn preventing the development of tumour cells into metastasis but the mechanisms are very poorly defined. Here we find that the expression level of miR-296-3p is much lower in the non-metastatic human prostate cancer (PCa) cell line P69 than that in the highly metastatic cell line M12, which is derived from P69. We demonstrate that miR-296-3p directly targets and inhibits the expression of intercellular adhesion molecule 1 (ICAM-1) in the malignant M12. The data from clinical tissue microarrays also show that miR-296-3p is frequently upregulated and ICAM-1 is reversely downregulated in PCa. Interestingly, ectopic expression of miR-296-3p in P69 increases the tolerance to NK cells whereas knockdown of miR-296-3p in M12 reduces the resistance to NK cells, which both phenotypes can be rescued by re-expression or silencing of ICAM-1 in P69 and M12, respectively. These results are also manifested in vivo by the decrease in the incidence of pulmonary tumour metastasis exhibited by knockdown of miR-296-3p in M12 when injected into athymic nude mice via tail vein, and consistently down-expression of ICAM-1 reverses this to increase extravasation of CTCs into lungs. Above results suggest that this newly identified miR-296-3p-ICAM-1 axis has a pivotal role in mediating PCa metastasis by possible enhancing survival of NK cell-resistant CTC. Our findings provide novel potential targets for PCa therapy and prognosis.

Cell Death and Disease (2013) 4, e928; doi:10.1038/cddis.2013.458; published online 21 November 2013

Subject Category: Cancer

Prostate cancer $(\mathrm{PCa})$ is the most common solid malignant tumour in men, and it ranks second overall in terms of mortality in developed countries. ${ }^{1}$ At least $90 \%$ of mortality from $\mathrm{PCa}$ is due to metastases rather than the primary tumours. ${ }^{2}$ The invasion-metastasis cascade involves a multistep cell-biological process, ${ }^{3}$ which is influenced by host and tumour molecular characteristics. During this process, mesenchymal tumour-like cells are highly mobile and enter quickly adjacent structure (intravasation), from where they travel through lymphatic and blood vessels as circulating tumour cells (CTC), which are single cells with malignant potential detected in the peripheral bloodstream and essential for establishing metastasis. ${ }^{4}$ CTC successfully achieve metastasis, which must perform well in multiple events, ${ }^{5,6}$ especially succeed in survival in the circulation. Most of CTC shed from the primary tumour site die during transport in vessels because of haemodynamic shear forces, angiogenic factors (such as blood platelets) ${ }^{6}$ and assault from immune effector cells. Natural killer (NK) cells are highly responsible for destroying CTC before their extravasation, ${ }^{7}$ whereas they exert only a minimal effect on both primary tumours and established metastases. However, the mechanisms underlying tumour cells escaping from NK cell lysis are still not understood.

Among of cell adhesion molecules (CAMs), intercellular adhesion molecule 1 (ICAM-1) is required for the $\beta 2$ integrin lymphocyte function associated antigen 1 (LFA-1)-dependent early stimulatory signal for NK cell cytotoxicity. ${ }^{8}$ It has been reported that the shed soluble ICAM-1 from melanoma cells to avoid surveillance of NK cells, ${ }^{9}$ however, whether ICAM-1 is involved in regulation of CTC resistance to NK cells and metastasis is poorly defined.

Various tumours overexpress some microRNAs (miRNAs) targeting $3^{\prime}$ untranslated region ( $3^{\prime}-U T R$ ) of main activating ligand genes such as MICA, MICB ${ }^{10}$ and ULBP $1^{11}$ to avoid immune recognition. For instances, miR-222 and miR-339 suppress ICAM-1 expression thereby downregulating the susceptibility of glioma cells to cytotoxic T-lymphocyte (CTL)mediated cytolysis, ${ }^{12}$ and miR-221-mediated translational repression of ICAM-1 on cholangiocytes gives a significant

\footnotetext{
${ }^{1}$ Department of Biochemistry and Molecular Cell Biology, Shanghai Key Laboratory of Tumor Microenvironment and Inflammation, Shanghai Jiao Tong University School of Medicine, Shanghai, China; ${ }^{2}$ Department of Oncology, No. 3 People's Hospital Affiliated to Shanghai Jiao-Tong University School of Medicine, Shanghai, China and ${ }^{3}$ State Key Laboratory of Oncogenes and Related Genes, Shanghai Jiao Tong University School of Medicine, Shanghai, China

${ }^{*}$ Corresponding author: J Yu, Department of Biochemistry and Molecular Cell Biology, Shanghai Jiao Tong University School of Medicine (SJTU-SM), 280 South Chongqing Road, Shanghai 200025, China. Tel: +86 21 54660870; Fax: +86 21 64661525; E-mail: Jianxiu.Yu@ gmail.com

Keywords: NK cells; ICAM-1; prostate cancer; miR-296-3p; metastasis

Abbreviations: PCa, prostate cancer; NK, natural killer; CTC, circulating tumour cell; EMT, epithelial-mesenchymal transition; ICAM-1, intercellular adhesion molecule 1; LFA-1, lymphocyte function associated antigen 1; LAMP-1, lysosomal-associated membrane protein-1; IFN- $\gamma$, interferon $\gamma$; TNF- $\alpha$, tumour necrosis factor $\alpha$; miRNA, microRNA; $3^{\prime}$-UTR, $3^{\prime}$ untranslated region; Calcein-AM, calcein acetyoxymethyl ester; mAb, monoclonal antibody

Received 28.8.13; revised 06.10.13; accepted 21.10.13; Edited by G Melino
} 
influence on the adherence of co-cultured T cells. ${ }^{13}$ Although these studies indicate that miRNAs mediate tumour cells evading from immune system monitoring, whether miRNAs influence tumour metastatic potential in vivo by escaping from NK cell lysis remains unclear.

In this study, we try to answer above questions and to explore why the metastatic potential of $\mathrm{PCa}$ is associated with their susceptibility to destruction of NK cells. ${ }^{7}$ We identify a new miRNA-296-3p-ICAM-1 axis has crucial roles in avoidance of CTC destruction by NK cells, thereby enhancing CTC survival and concomitantly promoting $\mathrm{PCa}$ metastatic extravasation.

\section{Results}

Characterization of human PCa cell lines P69 and M12.

P69 is an immortalized, low-tumourigenic, non-metastatic prostate epithelial cell line, ${ }^{14}$ whereas highly tumourigenic and metastatic M12 is derived from P69 and mainly contains a deletion of 19q13.1->19pter. ${ }^{15}$ We first used the xCELLigence RTCA-DP System real-time monitoring the migration curves of P69 and M12. The impedance increase correlates to increasing numbers of migrated cells. ${ }^{16,17}$ P69 displayed a flat line in cell index of migration; in contrast, M12 exhibited a strong migration curve tending to upward in $24 \mathrm{~h}$ (Figure 1a). This suggests that P69 has a very low motility capacity while M12 endows with the high motility ability.
Consistent with above, 3D culture assays displayed morphologic changes that defined different tumourigenic and metastatic characteristics of these two cell lines. P69 produced acini morphology whereas M12 displayed a highly disorganized mass of cells and star-like morphology (Figure 1b). The loss of E-cadherin is a hallmark of epithelialmesenchymal transition (EMT) and coincides with the transition from well-differentiated adenoma to invasive carcinoma. ${ }^{18}$ Thus, immunostaining for the mesenchymal marker Vimentin and the epithelial marker E-cadherin was conducted to observe the 3D culture morphologic structures. P69 displayed almost no expression of Vimentin but abundant E-cadherin; conversely, M12 showed high Vimentin but loss of E-cadherin (Figure 1c). This was confirmed by flow cytometric analysis (Figure 1d). Collectively, these results indicate that these two cell lines are very different in metastatic potential and can be used for the following studies.

P69 is more sensitive to ex vivo-expanded human NK cell-mediated cytolysis than M12. During the transformation process from $\mathrm{P} 69$ to $\mathrm{M} 12$, which is obtained by sequential passages in male athymic nude mice,${ }^{15}$ cancer cells might undergo escaping from immune effector cells especially NK cells in blood vessels. Human NK cells were purified and ex vivo expanded as described previously. ${ }^{19,20}$ We examined the expression levels of receptors on these NK cells showing a highly activated NK cell receptor expression
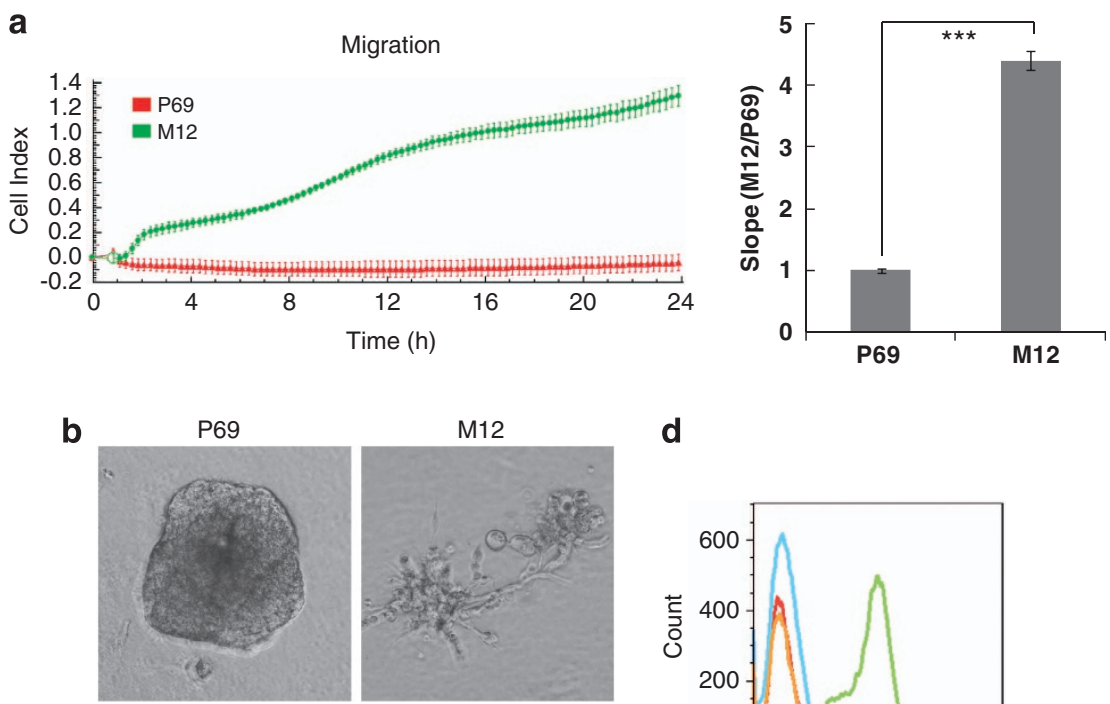

d

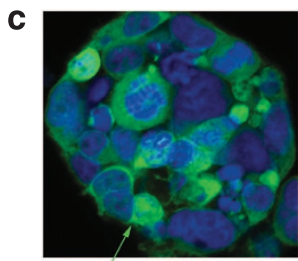

E-cadherin

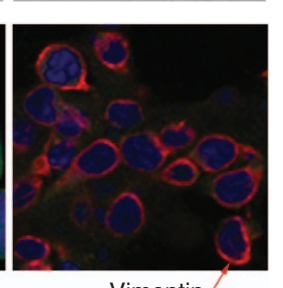

Vimentin

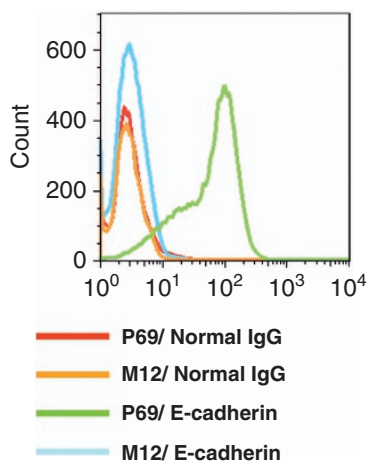

Figure 1 Morphological and metastatic differences between P69 and M12. (a) Migration kinetics of P69 and M12, as shown by real-time monitoring of live cell migration (P69-red, M12-green). (b) Light microscopy images of P69 and M12 were taken from cultures grown in 3D culture matrix. Magnification, $\times 20$. (c) Immunofluorescence staining of P69 and M12 grown in 3D Culture Matrix (Vimentin-red, E-cadherin-green, DAPI-blue). (d) The expression levels of E-cadherin in P69 (green line) and M12 (blue line) were detected by flow cytometry. IgG isotype antibody was used as a negative control 
pattern, which was characterized by high expressions of NKG2D and CD226 (DNAM-1), and moderate expressions of natural cytotoxicity receptors and low expressions of inhibitory receptors (Supplementary Figure S1).

To verify whether there is different immune response between P69 and M12, we performed calcein acetyoxymethyl ester (calcein-AM) cytotoxicity assays to evaluate the activities of ex vivo-expanded human NK cells against P69 and M12. P69 was more sensitive to NK cells compared with M12 at three different ratios of effector/target (E/T; Figure 2a). The degranulation assay based on detecting the expression level of lysosomal-associated membrane protein-1 (LAMP-1, also named as CD107 $\alpha$ ) on NK cell surface also showed higher activities of NK cells against P69 than those of M12 (Figure 2b). NK cell activation is often marked by polarization of perforin-containing cytotoxic granules toward target cells, thus we used immunofluorescence staining to evaluate perforin polarization of NK cells against P69/M12, and found more perforin-containing granules polarized toward P69 compared with M12 (Figure 2c; Supplementary Figure S2). Furthermore, conjugation assays were conducted to show that conjugate formation between NK cells with P69 was higher than that with $\mathrm{M} 12$ (Figure 2d), suggesting there are the differential expressions of ligands on P69/M12, thereby leading to different NK cell cytolytic activities.

The differential expressions of ligands cause the different NK cell susceptibility between P69 and M12.

As several studies have suggested some tumour cells evade immune surveillance by secreting some special soluble molecules such as soluble $\operatorname{ULBP}^{21}$ and soluble ICAM- $1,{ }^{9}$ we wanted to confirm whether there are some different soluble molecules secreted from these two cells mediating the different susceptibility of P69/M12 to NK cells. We performed cytotoxicity by calcein-AM assay to find no significant difference in cytotoxicities among NK cells against P69 or M12 cells supplemented with the medium supernatants harvested from P69 and M12 cultured for $48 \mathrm{~h}$, respectively, indicating that the different susceptibility is indeed not due to soluble molecules secreted from these two target cells (Figure 2e). In addition, we did not observe significant difference in secretion of interferon $\gamma($ IFN $-\gamma)$ and tumour necrosis factor $\alpha($ TNF- $\alpha)$ from NK cells cultured with P69 or M12 in a relatively short period (Supplementary Figure S3). Thus, above results suggest that M12 is more resistant to NK cells than P69 and this differential susceptibility is caused by the differential expressions of ligands on cell surface, but not by either soluble molecules secreted from these two cancer cells or cytokines from NK cells.

ICAM-1 mainly contributes to the differential NK cell susceptibility between P69 and M12. Next, to find specific ligands on P69 and M12 are involved in their differential NK cell cytotoxicities, we analyzed the expression levels of ligands on P69/M12 (Figure 3a) and found that ICAM-1 and ULBP2 (one of ligands of NKG2D) were expressed higher in P69 than those in M12, indicating that ICAM-1 and ULBP2 may be key factors involved in the differential NK cell susceptibility.
Binding of LFA-1 to ICAM-1 on target cell initiates activation signals for NK cell cytotoxicity, contributing to strong adhesion to target cell and polarization of cytolytic granules in NK cells. $^{8,22}$ To verify contribution of LFA-1/ICAM-1 to the differential NK cell cytotoxicities between P69 and M12, we knocked down ICAM-1 by shRNA-based lentiviral system in P69 (P69 shICAM-1) and overexpressed ICAM-1 in M12 (M12 ICAM-1) (Supplementary Figures S4 and S5) to test changes in NK-target conjugate formation and cytotoxicity. The results of three assays including calcein-AM cytotoxicity (Figure $3 b$ ), conjugate formation (Figure 3c) and degranulation (Figure 3d) showed that down-expression of ICAM-1 in P69 markedly decreased the NK cell susceptibility of P69 while up-expression of ICAM-1 in M12 significantly increased the NK cell susceptibility of M12. As expected, blockade of the receptor of ICAM-1, LFA-1 by a specific monoclonal antibody $(\mathrm{mAb})$ led to remarkable reduction of cytotoxicity (Figure 3e), suggesting that LFA-1/ICAM-1 greatly contributes to NK cell cytolysis of P69/M12. Considering the importance of NKG2D in NK cell recognizing and killing, we blockaded it with the specific $\mathrm{mAb}$ and also found inhibition of NK cell killing against both P69 and M12 (Supplementary Figure S6). This indicated LFA-1/ICAM-1 could be co-stimulatory for NKG2D-dependent NK cell cytotoxicity.

MiR-296-3p inhibits ICAM-1 by directly targeting its $\mathbf{3}^{\prime}$-UTR. To find out miRNAs involved in tumour cell resistance to NK cell, we sequenced the small RNA transcriptomes of P69/M12 by using the Illumina high-throughput sequencing technology, and chose some miRNAs as candidates, which were differentially expressed in between P69 and M12, to establish stable cell lines ectopically expressing 17 miRNAs by lentiviral system, respectively. We performed the calceinAM assay for above cell lines, and found only ectopic expression of miR-296-3p in P69 significantly reduced the NK cell susceptibility (Figure $4 a$ ).

The sequencing data showed that miR-296-3p in M12 was expressed much higher than that in P69. Conversely, ICAM-1 transcription in M12 were lower than that in P69 (Figure 4b). Combined with the prediction by miRanda-mirSVR, ${ }^{23}$ these data indicated that miR-296-3p may target ICAM-1 gene. Indeed, the quantitative-PCR confirmed that the expression level of miR-296-3p in M12 exhibited about 2.5-fold higher than that in P69 (Figure 4c); and in agreement with this, western blotting and immunofluorescence staining also showed that the expression level of ICAM-1 protein in M12 was lower than that in P69 (Figure 4d; Supplementary Figure S7). Thus, these results suggest that miR-296-3p potentially targets ICAM-1 gene.

To verify whether miR-296-3p targets the $3^{\prime}$-UTR of $I C A M-1$, we performed the dual luciferase reporter assay. The ICAM-1 $3^{\prime}$-UTR was cloned into the vector psiCHECK-2 and the putative miR-296-3p binding site mutant was also generated (Figure 4e). The wild-type (WT) or mutant reporter construct with the plasmid expressing miR-296-3p or control vector was transfected into HEK-293T. The luciferase activity of the WT reporter was significantly inhibited by exogenous expression of miR-296-3p, whereas the luciferase activity of the mutant reporter was not affected by miR-296-3p (Figure 4f). Furthermore, we also transfected miRZip shRNA 
a

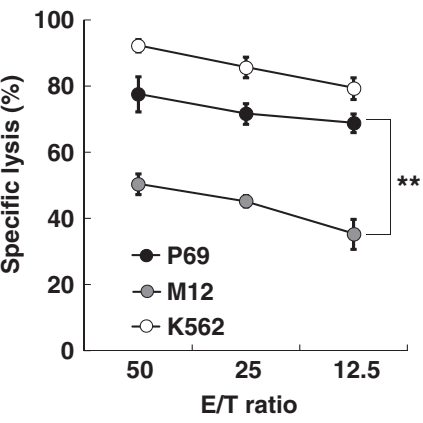

b
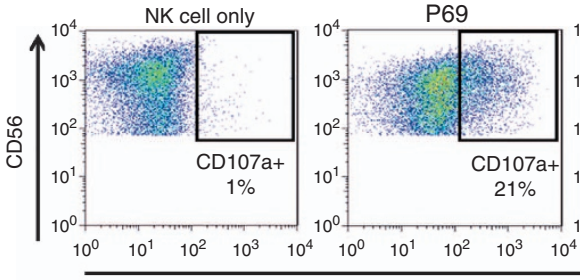

CD107a

d
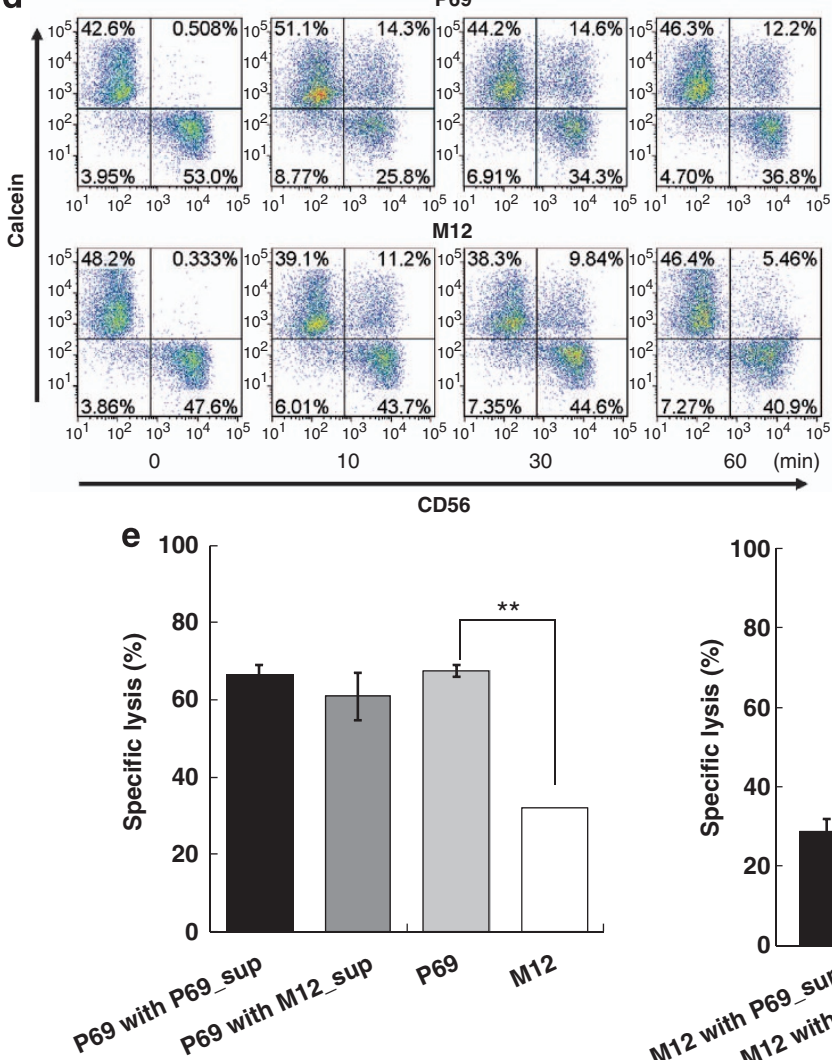

C

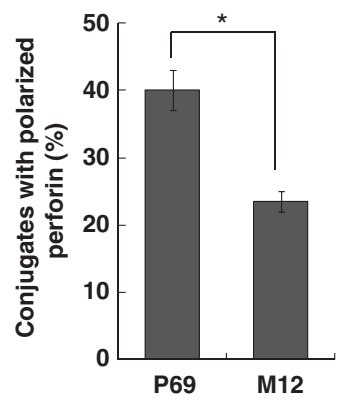

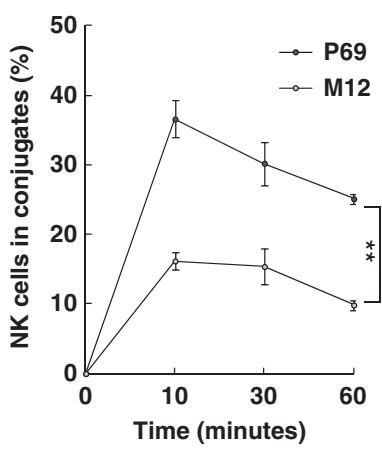

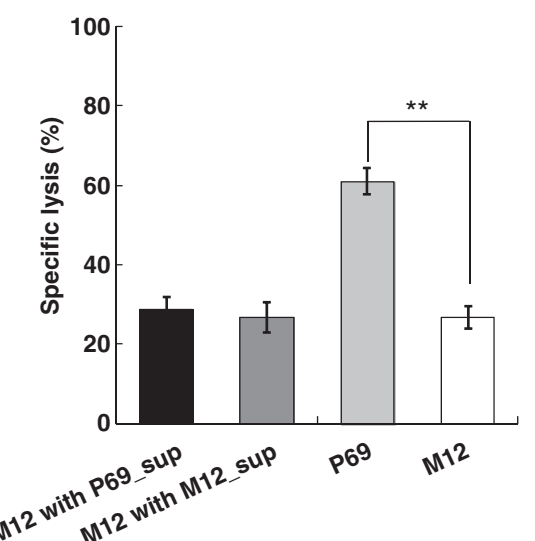

Figure 2 P69 are more susceptible to NK cells than M12. (a) Human NK cell cytotoxicity to P69, M12 and K562 (as a positive control) was assessed by calcein release assays in various $\mathrm{E} / \mathrm{T}$ ratios as described in Materials and Methods section. Data are the mean \pm S.E.M. of three independent experiments. (b) The mixtures of NK cells and $\mathrm{P} 69$ or M12 at an E/T ratio of $1: 2$ were incubated at $37^{\circ} \mathrm{C}$ for $2 \mathrm{~h}$ and then stained for CD107 $\alpha$ and CD56. The gated on CD56-posivtive cells were analyzed and the numbers in boxes indicated percentage of CD107 $\alpha$-positive NK cells. The data are representative of three separate experiments (left graph). Data were the mean \pm S.E.M. of three independent experiments (right panel). (c) Polarization of perforin-containing granules in NK cells against P69 or M12 were expressed as percentage of NK cells showing polarized perforin. Data are the mean \pm S.E.M. from three independent experiments. (d) The conjugate formation of NK cells with P69 or M12 at indicated time points was identified by flow cytometry. The gated on CD56-posivtive cells were analyzed and the numbers in different quadrants indicated percentage of cells. One representative experiment out of three was shown (left graph). The fraction of two-colour NK cells was analyzed and data are the mean \pm S.E.M. of three independent experiments (right panel). (e) P69 (left panel) or M12 (right panel) was supplemented by the media harvested from 48-h cultural supernatants of P69 or M12 cultures and then calcein release assay was performed at an $E / T$ ratio of 5-1. Data are the mean \pm S.E.M. of three independent experiments 
a
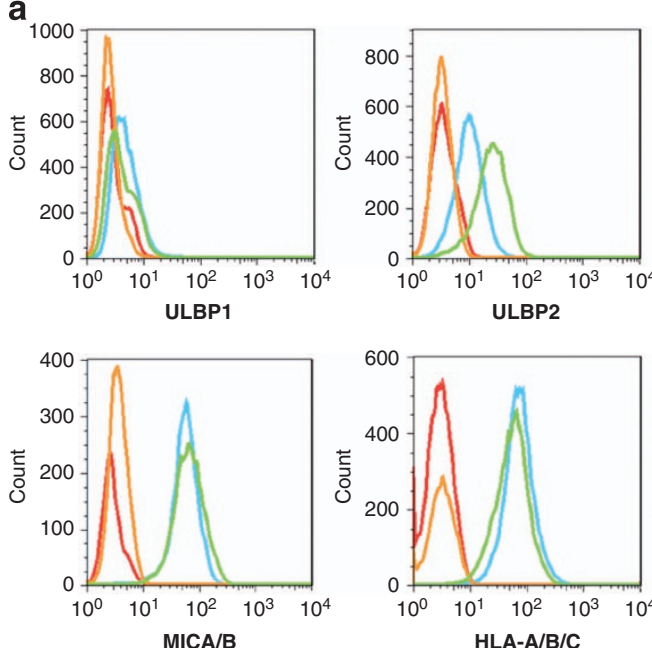

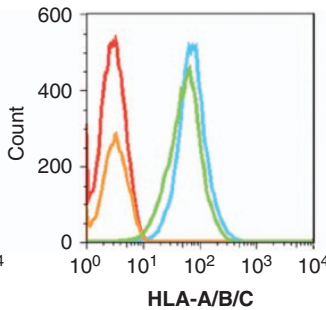

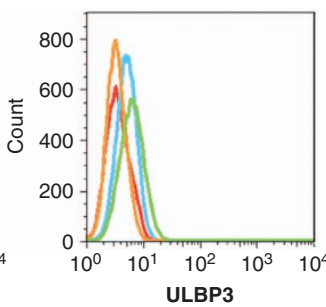
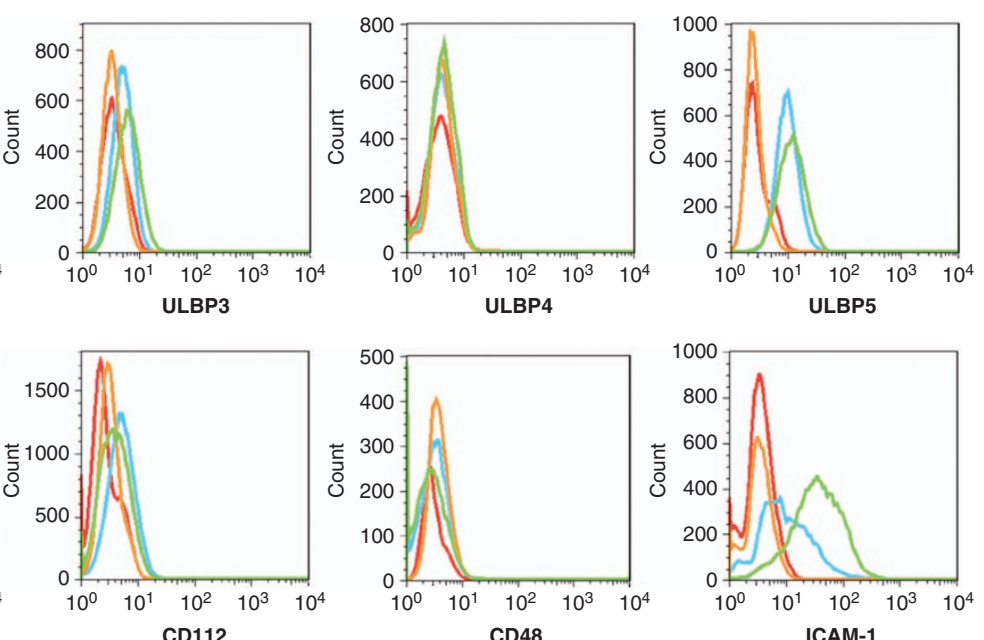

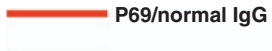

$\mathrm{P} 69 /$ specific antibody

M12/normal IgG

M12/specific antibody

b

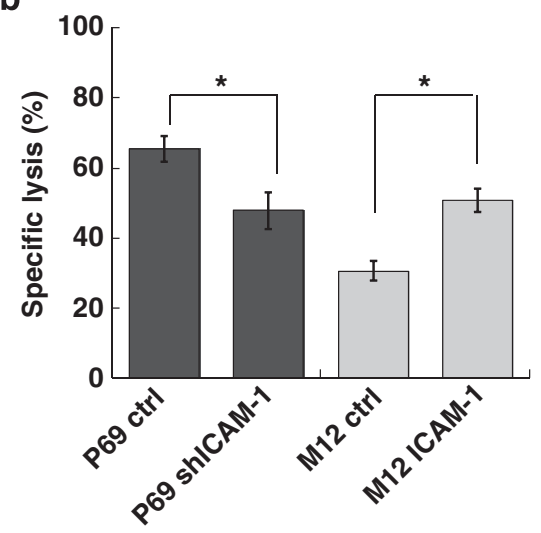

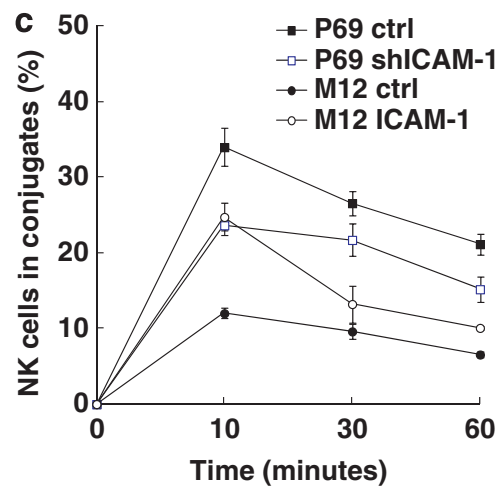

Time (minutes)
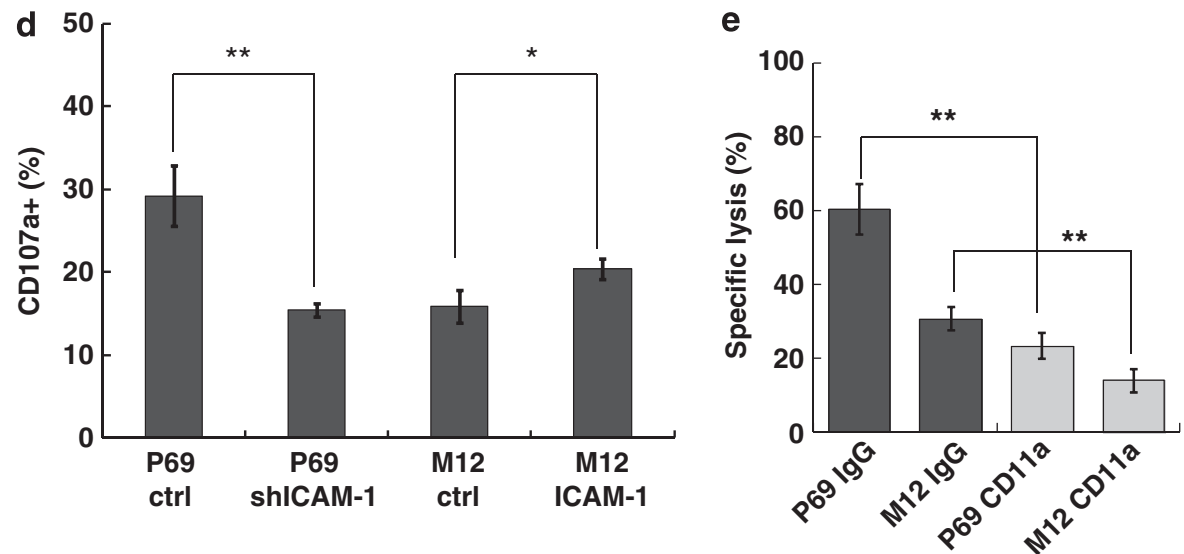

Figure 3 ICAM-1 contributes to the differential susceptibility of P69 and M12 to NK cells. (a) The expression levels of ligands on P69 (green lines) or M12 (blue lines) paired to NK cell receptors were analyzed by flow cytometer. Red and orange lines represent P69 and M12 with PE or APC-conjugated isotype matched IgG as negative controls, respectively. (b) The NK cell cytotoxicities to ICAM-1-knockdown P69 (P69 shICAM-1) and ICAM-1-overexpressing M12 (M12 ICAM-1) were assessed by calcein release assay. (c) The conjugation of NK cell to P69 shICAM-1 and M12 ICAM-1 were determined. Data are the mean \pm S.E.M. from three independent experiments. (d) The degranulation of NK cells against P69 shICAM-1 and M12 ICAM-1 was performed. Data are the mean \pm S.E.M. of three independent experiments. (e) NK cells were incubated with anti-CD11 $\alpha$ (a subunit of LFA-1) mAbs to block function of LFA-1 or isotype-matched IgG as a control for 30 min and then mixed with P69 or M12 at an E/T ratio of 5-1 for calcein release assay. Data are the mean \pm S.E.M. of three independent experiments 


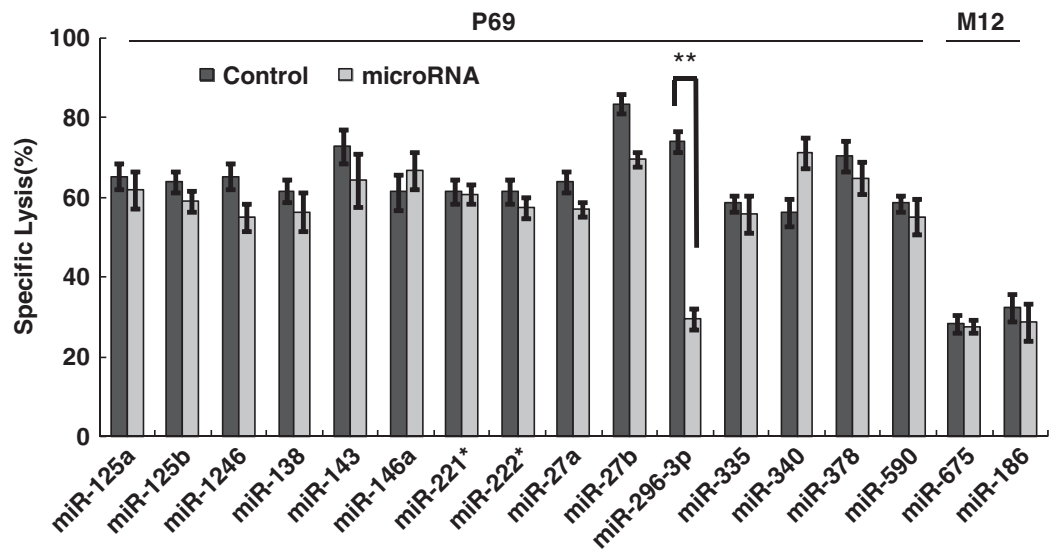

b

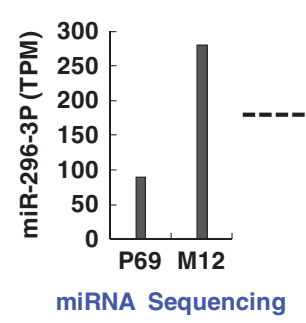

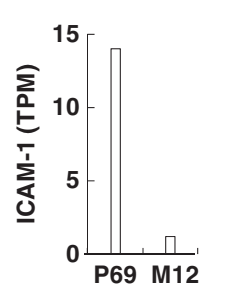

DGE Sequencing e

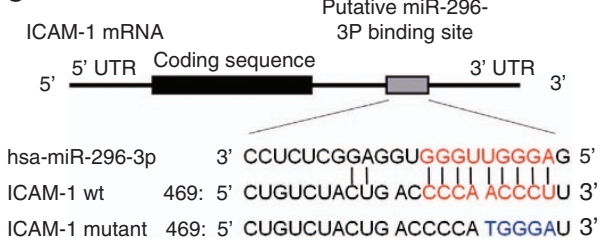

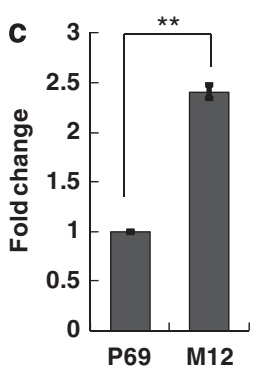

d

kDa

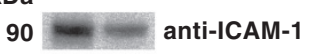

43 $6^{9} n^{2}$

$\mathbf{f}$

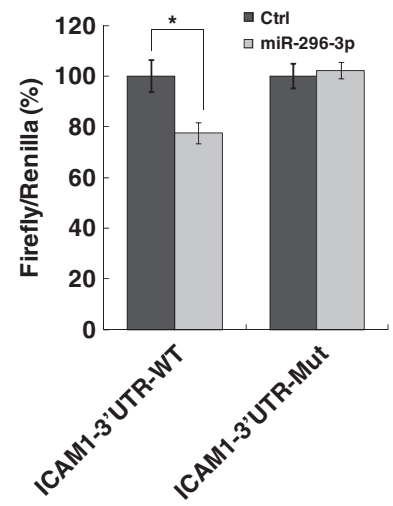

$\mathbf{g}$

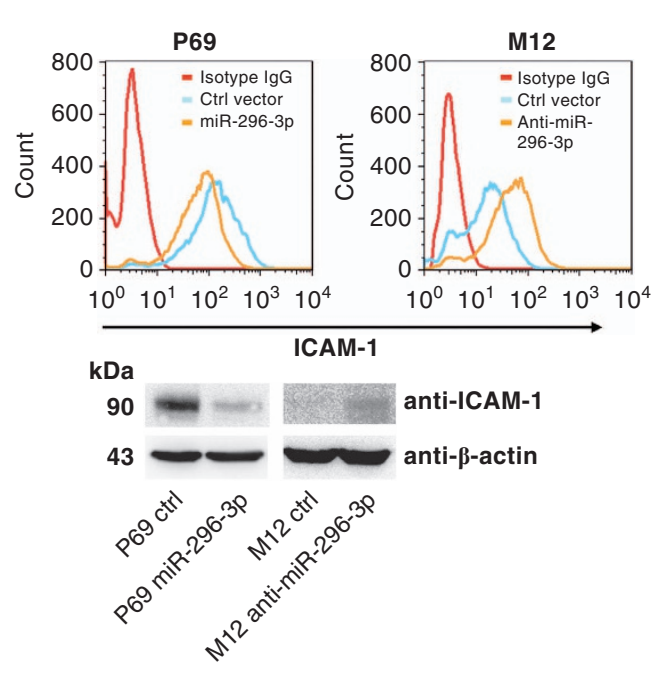

Figure 4 MiR-296-3p directly regulates ICAM-1 in PCa cells. (a) Screening of candidate miRNAs (in target cells) involved in the resistance to NK cells were performed using calcein release assay. Data are the mean \pm S.E.M. of three independent experiments. (b) The expression level of miR-296-3p and transcription level of ICAM-1 in P69 and M12 were analyzed by miRNA transcriptome sequencing and DGE sequencing, respectively. TPM means transcripts per million. (c) Real-time PCR in $\triangle \triangle C t$ method was performed to identify the expression levels of miR-296-3p in P69 and M12. One representative experiment out of three was shown. (d) The protein levels of ICAM-1 in P69 and M12 were determined by western blotting. One representative experiment out of three was shown. (e) The predicted miR-296-3p binding site (red) and their mutant (blue) in the $3^{\prime}$-UTR of ICAM-1 mRNA are indicated. Number shows position of nucleotides in the $3^{\prime}$-UTR of ICAM-1 mRNA. (f) HEK-293T cells were transfected with psiCHECK-2 containing WT or mutant ICAM-1 3'-UTR and miR-296-3p or mock control vector. The Renilla luciferase activity was normalized on the constitutive activity of firefly luciferase. Data are the mean \pm S.E.M. of three independent experiments. (g) The expression of membrane-bound or total ICAM-1 in P69 and M12 were analyzed by flow cytometry (upper panel) and western blotting (bottom panel), respectively. One representative experiment out of three was shown

for silencing of miR-296-3p in M12 by lentiviral infection, getting another pair of stable cell lines M12-ctrl and M12anti-miR-296-3p. Results from both flow cytometry and immunoblotting showed that ICAM-1 protein was downregulated in P69-miR-296-3p and reversely upregulated in M12-antimiR-296-3p (Figure 4g), suggesting that miR-296-3p acts as a 
suppressor of ICAM-1. Therefore, above results provide the first evidence that miR-296-3p directly inhibits ICAM-1 gene expression by targeting its $3^{\prime}-$ UTR.

MiR-296-3p is upregulated and negatively correlated with ICAM-1 in human PCa tissues. To extend our analysis to clinical relevance, 40 of human PCa tissues and 32 of prostate normal tissues were stained with the specific miR-296-3p probe tagged with DIG labelling for in situ hybridization (ISH) analysis (Figure 5a). As expected, the expression levels of miR-296-3p were high in about $40 \%$ of cancer tissue samples and middle in approximately $25 \%$ of cancer tissue samples. Conversely, about $90 \%$ of normal tissue samples expressed low levels of miR-296-3p and almost $10 \%$ expressed middle levels of miR-296-3p (Figure 5b). This data supported our hypothesis that miR296-3p has an onco-miRNA role.

These tissue arrays were also stained with anti-ICAM-1 $\mathrm{mAb}$ for immunohistochemical (IHC) analysis (Figure $5 \mathrm{c}$ ). Unlike the results of ISH, ICAM-1 was expressed heterogeneously in $\mathrm{PCa}$ tissues. Nevertheless, ICAM-1 was expressed at low (below $5 \%$ of positive area/total area) in almost $90 \%$ of normal specimens. Most importantly, we observed a significant negative correlation between the expression levels of ICAM-1 and miR-296-3p in PCa tissues, Spearman $r=-0.4365, P=0.0055, n=39$ (Figure $5 \mathrm{~d}$ ). This result suggests that miR-296-3p negatively regulates the expression of ICAM-1 in tissues. a

Normal tissues
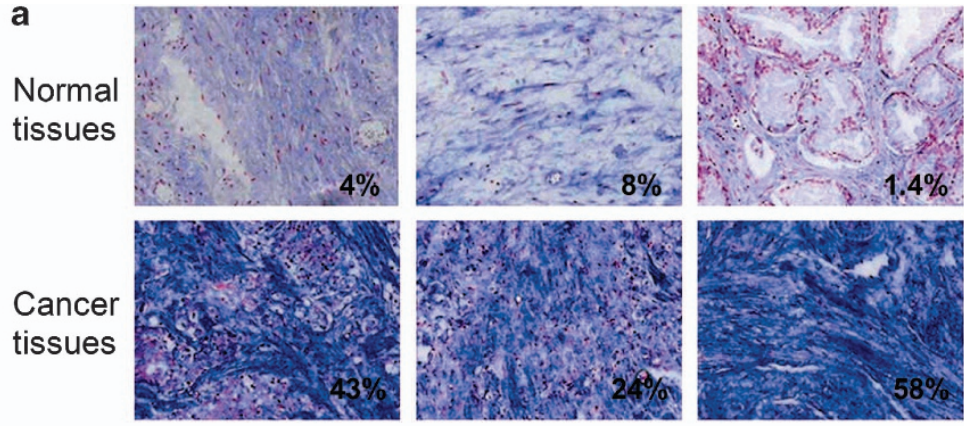

b

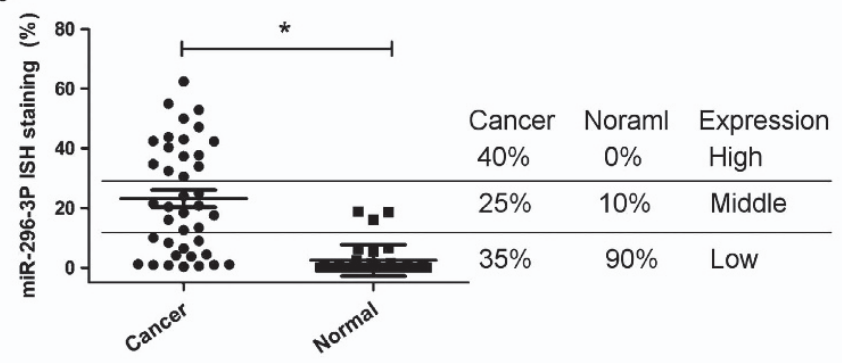

c

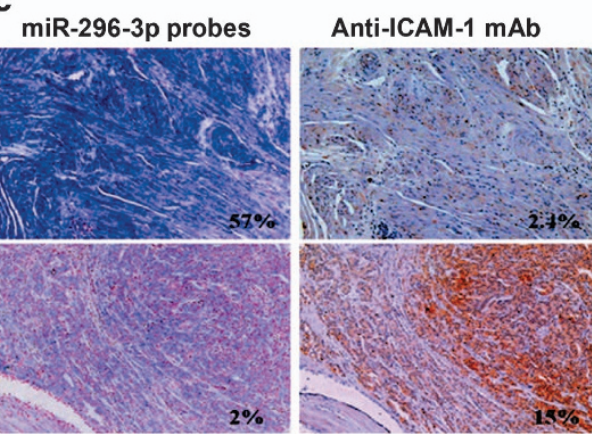

d

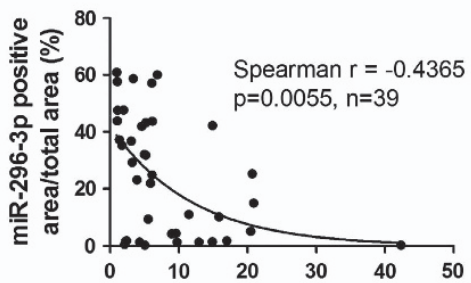

ICAM-1 positive area/total area(\%)

Figure 5 MiR-296-3p is upregulated and negatively correlated with ICAM-1 in human PCa tissues. (a) The expression levels of miR-296-3p in human prostate normal tissues (upper panel) and cancer tissues (bottom panel) were detected by ISH as described in Material and methods section. Representative staining tissue sections were presented and the numbers shown in images indicated the ratio of positive staining area. (b) Vertical coordinates indicated the percentage ratio of positive staining area to total tissue area and specimens were ranked into as high (upper $30 \%$ ), middle (a range of 10\%-30\%) and low (below 10\%) expression levels. Data were analyzed with the MannWhitney test to determine the statistic significance $(P<0.0001)$. (c) Human PCa tissue arrays were stained with DIG-conjugated probe for miR-296-3p (left column) or antiICAM-1 mAbs (right column). Representative staining tissue sections were presented and the numbers shown in images indicated the ratio of positive staining area to total tissue area. (d) The expression correlation between miR-296-3p and ICAM-1 in PCa tissue was analyzed with the method of Spearman, the coefficient values: $r=-0.4365$, $P=0.0055, n=39$ 
MiR-296-3p increases tumour cell resistance to NK cell via downregulating the ICAM-1 expression. To verify whether LFA-1/ICAM-1 is essential for miR-296-3p in regulation of tumour cell resistance to NK cell, we performed the cytotoxicity assay following functional blockade of LFA-1 on NK cells by a specific anti-CD $11 \alpha \mathrm{mAb}$, and observed the similar low NK cell cytotoxicities against four tumour cell lines P69-ctrl, P69-miR-296-3p, M12-ctrl and M12-anti-miR-296$3 p$, although ectopic expression of miR-296-3p in P69 reduced or knockdown of miR-296-3p in M12 increased the cytotoxic activities compared with those in the corresponding control cells when treated by normal IgG antibody (Figure 6a). This result suggests that miR-296-3p in regulation of tumour cell resistance to NK cell is mainly mediated by LFA-1/ICAM-1.

a

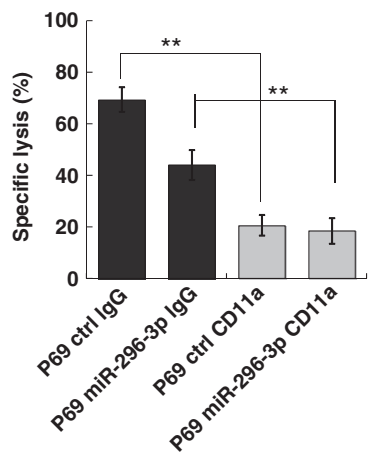

b
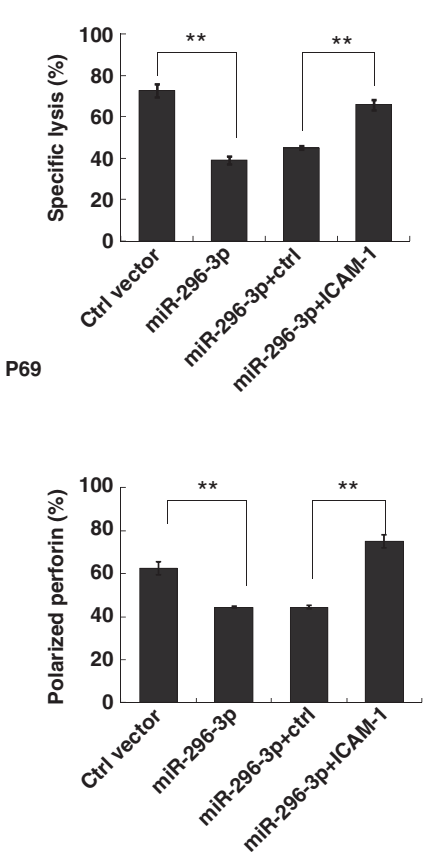
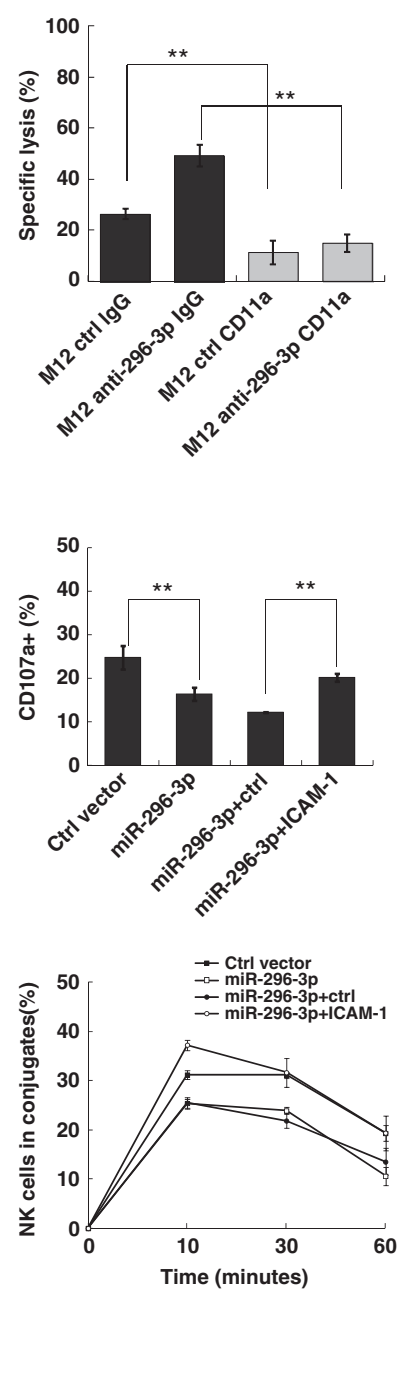

We next evaluated whether miR-296-3p-ICAM-1 axis has a critical role in mediating tumour cell resistance to NK cell. MiR-296-3p overexpression in P69 significantly led to the enhanced resistance to cytolysis, the decreased expression of CD107 $\alpha$ on NK cell surface, the less perforin polarization and the reduced NK-target conjugate formation compared with P69 transfected with control vector (Figure 6b). These results show that miR-296-3p can reduce the NK cell cytotoxicity against P69. To further reveal whether ICAM-1 contributes to miR-296-3p in regulation of tumour cell susceptibility to NK cell, we generated a stable cell line P69-miR-296-3p + ICAM-1 (Supplementary Figure S8, left) to show that ICAM-1 overexpression in P69-miR-296-3p completely reversed the reduced NK cell cytotoxicity (Figure 6b), suggesting that miR-296-3p enhances tumour

C
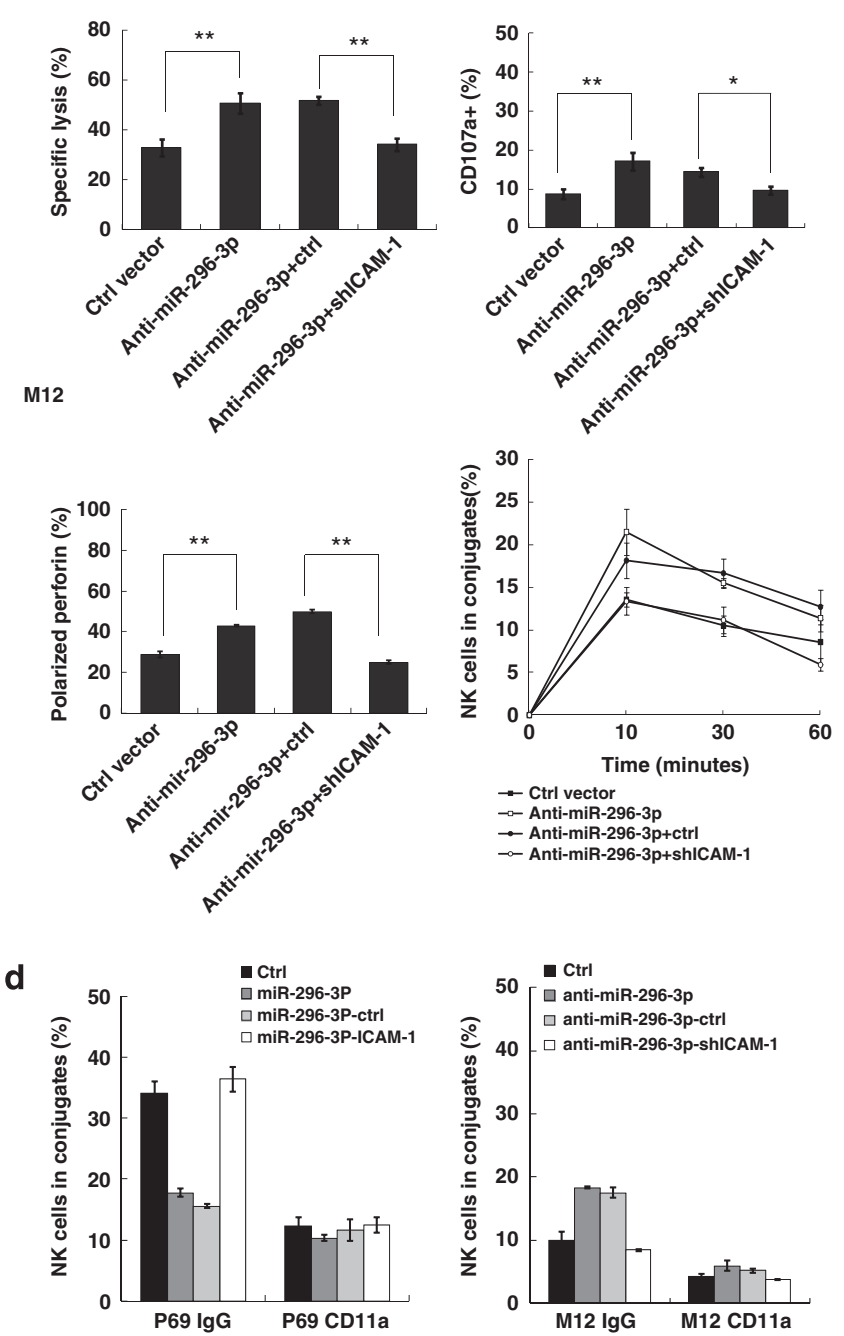

Figure 6 MiR-296-3p affects the sensitivity of PCa cells to NK cells. (a) NK cells were incubated with anti-CD11 $\alpha$ mAbs or isotype-matched lgG and then the cytotoxicity assay was performed against miR-296-3p-overexpressing P69 (P69 miR-296-3p) (left panel) and miR-296-3p-silencing M12 (M12 anti-miR-296-3p) (right panel). (b) The NK cell cytotoxicity (left upper panel), degranulation (right upper panel), perforin polarization (left bottom panel) and conjugate formation (right bottom panel) against miR-296-3p P69 and the ICAM-1-overexpressing miR-296-3p P69 (miR-296-3p + ICAM-1 P69) cells were performed as indicated. (c) The NK cell cytotoxicity (left upper panel), degranulation (right upper panel), perforin polarization (left bottom panel) and conjugate formation (right bottom panel) against the anti-miR-296-3p M12 and the ICAM-1-knockdown anti-miR-296-3p M12 (anti-miR-296-3p + shICAM-1 M12) cells were performed as indicated. (d) The conjugate formation between NK cells and P69 (left panel) or M12 (right panel) stable cell lines after blockade of CD11 $\alpha$ on NK cells with anti-CD11 $\alpha$ mAbs (isotype-matched lgG as a control) were examined as previously described. All above data were the mean \pm S.E.M. from three independent experiments 
cell resistance to NK cell by downregulating ICAM-1 expression.

Consistent with the above results, knockdown of miR-296-3p in M12 remarkably increased tumour cell susceptibility to NK cell compared with those in M12-ctrl cells, based on the above same four sets of experimental data (Figure 6c). As knockdown of miR-296-3p actually increased ICAM-1 expression (Figure 4g), we then down-expressed ICAM-1 by the similar RNA interference technique in M12-anti-miR-296-3p cells, getting in a stable cell line M12-anti-miR-296-3p + shICAM-1 (Supplementary Figure S8, right), which reversely reduced the NK cell cytotoxicities to the similar levels as those in M12-ctrl cells (Figure 6c).

To further support our hypothesis, we also performed NKtarget conjugation assay following blockade on LFA-1/CD11 $\alpha$ on NK cell by specific mAb, and observed that the conjugate formation was down to the similar low levels in all above established P69 or M12 stable cell lines (Figure 6d). Taken together, we concluded miR-296-3p downregulates ICAM-1 expression, thereby affecting LFA-1/ICAM-1 axis to increase tumour cell resistance to NK cell.

MiR-296-3p-ICAM-1-NK axis in control of pulmonary metastasis of M12 in vivo. To further determine the role of MiR-296-3p-ICAM-1 axis in vivo, we injected each group of $2.5 \times 10^{6} \mathrm{M} 12$ expressing the luciferase report gene into 8 -week athymic nude mice via tail vein. By following an established protocol, ${ }^{24}$ we monitored the pulmonary metastatic potential of tumour cells by living image system at 0,24 and $48 \mathrm{~h}$ after intravenous inoculation. At $0 \mathrm{~h}$, lung luminescence was observed initially comparable in all M12 variants (Figures $7 \mathrm{a}$ and b). At $24 \mathrm{~h}$, the intensity of lung luminescence in miR-296-3p-scilencing M12 was attenuated more than that in M12-ctrl cells (Figures 7a and b). At $48 \mathrm{~h}$, the lung luminescence in miR-296-3p-silencing M12 was almost undetectable whereas M12-ctrl still showed luminescent (Figures $7 \mathrm{a}$ and b). Moreover, knockdown of ICAM-1 in miR-296-3p-scilencing M12 cells exhibited similar levels of a
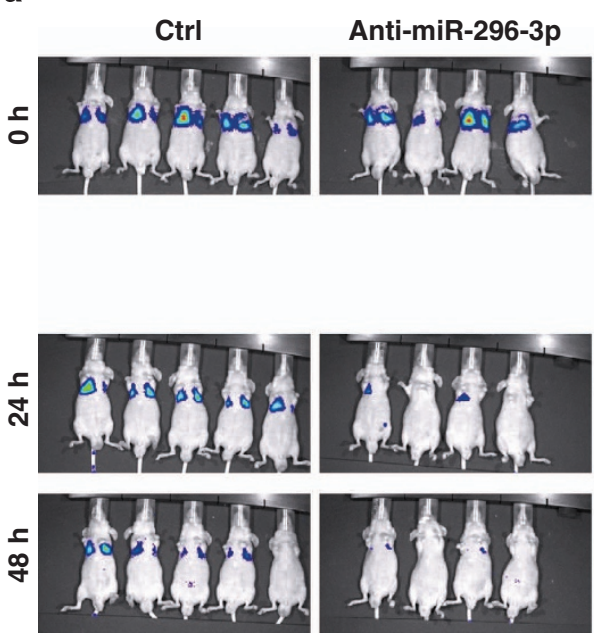

Anti-miR-296

$-3 p+c t r l$
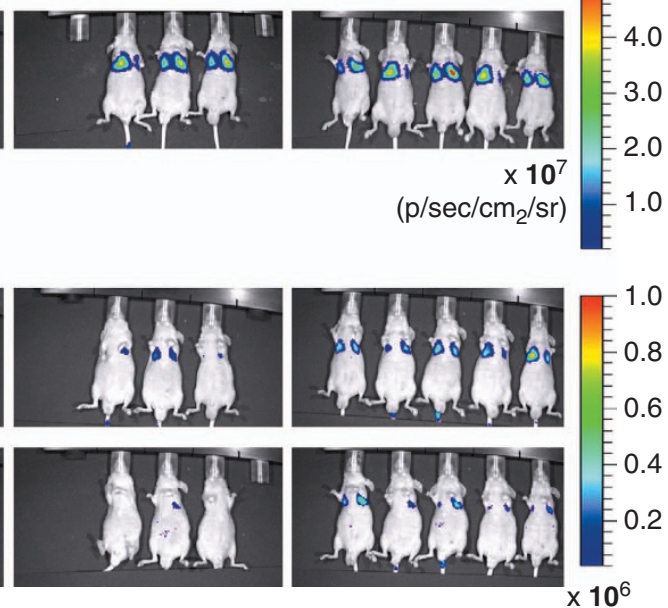

Anti-miR-296-3p+ shICAM-1

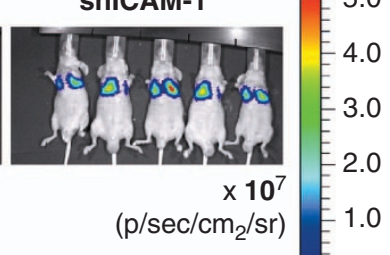

1.0

b

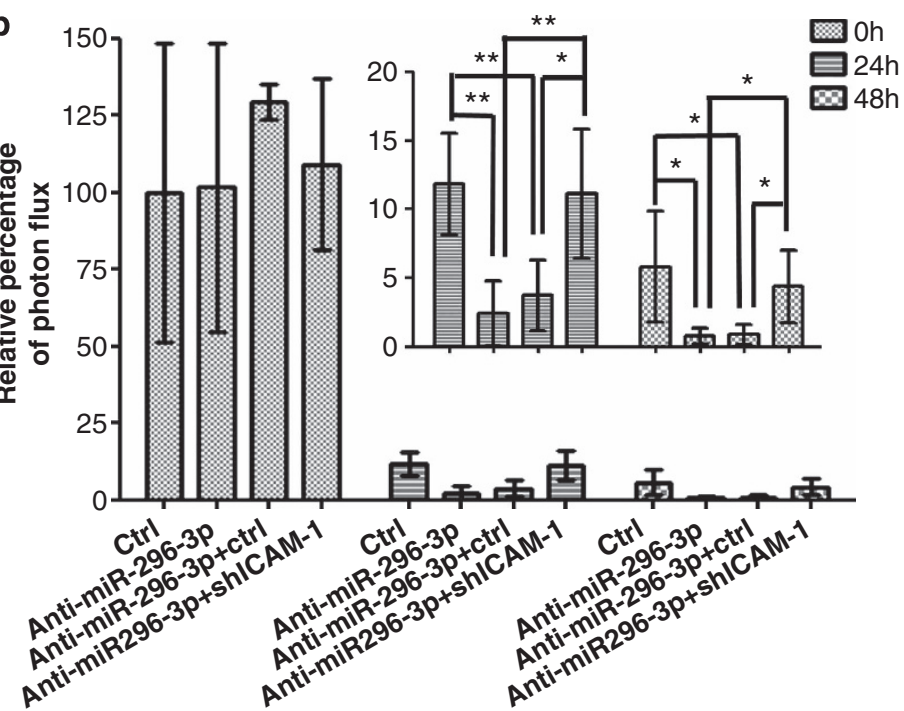

Figure 7 (Continued) 


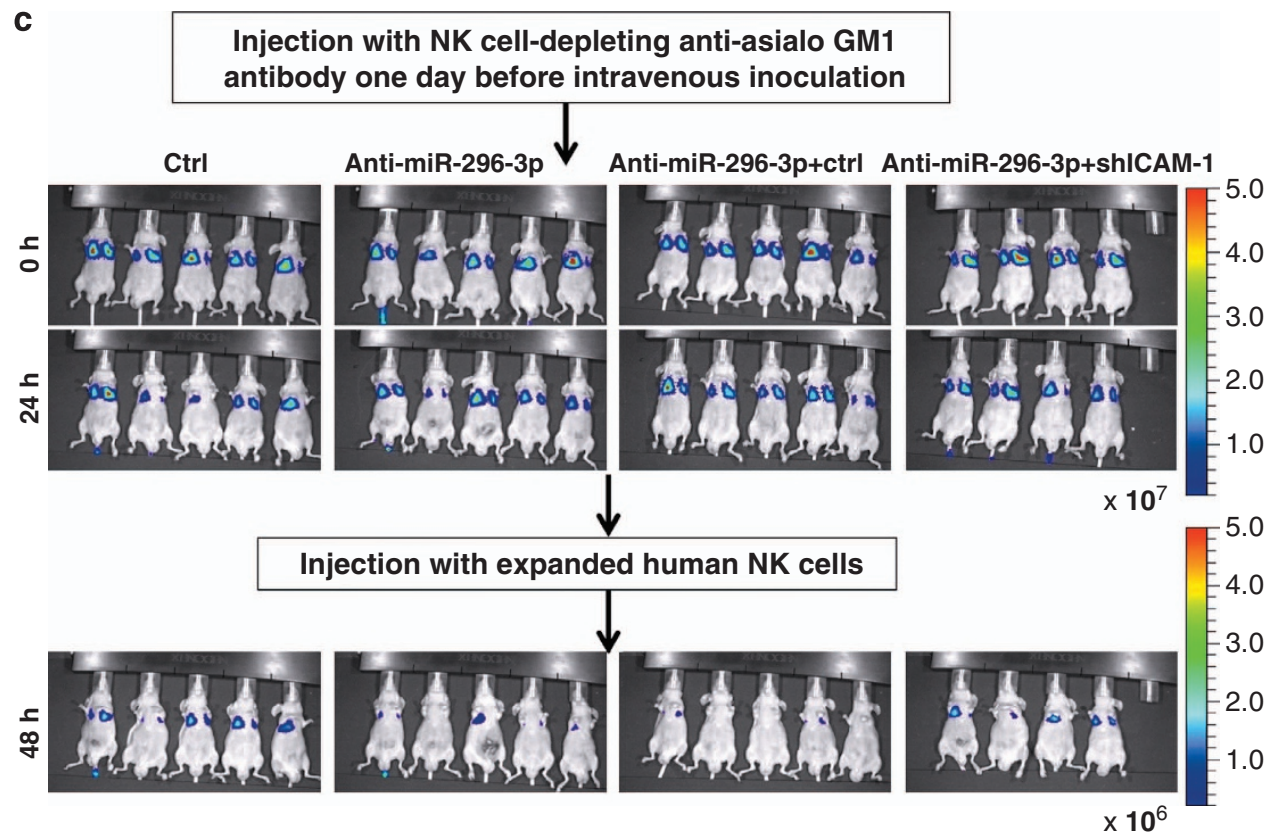

$\left(\mathrm{p} / \mathrm{sec} / \mathrm{cm}_{2} / \mathrm{sr}\right)$

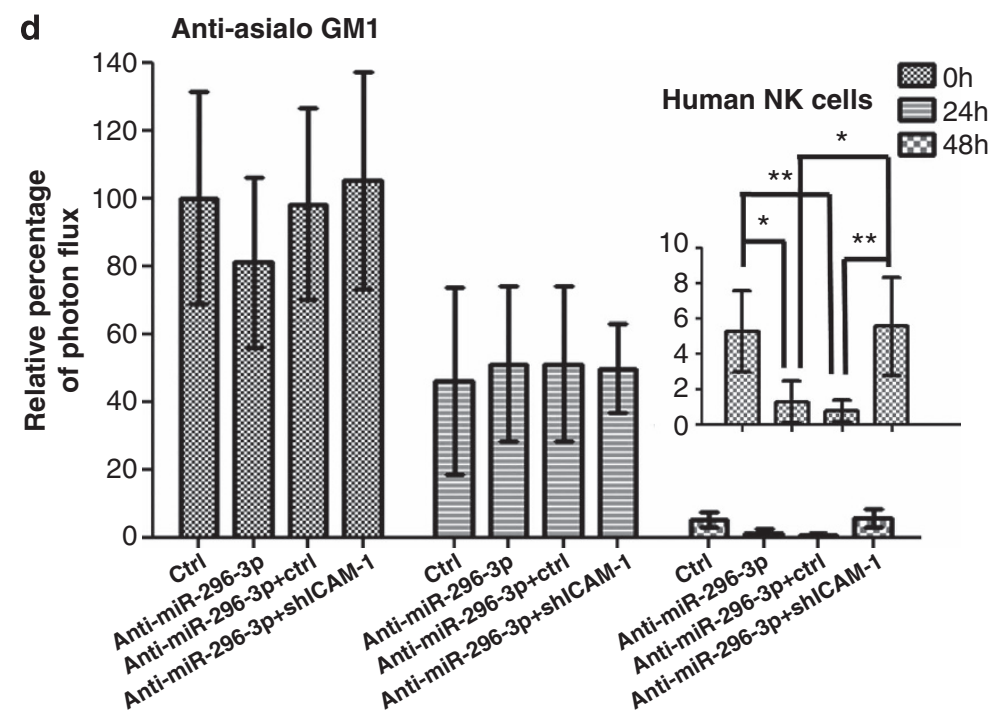

Figure 7 MiR-296-3p-ICAM-1-NK signalling axis in control of pulmonary metastasis of M12 in vivo. (a) BALB/C athymic mice (nu/nu) at 8 weeks old were injected intravenously with $2.5 \times 10^{6}$ of $\mathrm{M} 12^{\text {luc }}$. The transferred M12 $2^{\text {luc }}$ in lungs was detected by IVIS spectrum image system at 0,24 and $48 \mathrm{~h}$ after injection and presented visually with various colour spots based on different intensity of luminescence. (b) The relative amounts of transferred $\mathrm{M} 12^{\text {luc }}$ in lung were presented as percentage. The photon flux of M12 control cells at $\mathrm{Oh}$ was set as a common denominator, by which all other photon flux values of M12 variants at three time points were divided. The data were representative of two independent experiments (five nude mice per group) and expressed as mean \pm S.E.M. (c) BALB/c athymic mice (nu/nu) at 8 weeks old were injected intraperitoneally with $50 \mu \mathrm{l}$ of NK cell-depleting anti-asialo GM1 antibody 1 day before intravenous injection of $2.5 \times 10^{6}$ of M12/uc. At $24 \mathrm{~h}$ after injection of M12luc, $2.5 \times 10^{7}$ of expanded human NK cells were injected intravenously into nude mice. The transferred $\mathrm{M} 12^{\mathrm{Iuc}}$ in lungs were detected by IVIS spectrum image system at 0,24 and $48 \mathrm{~h}$, and (d) the data were processed and presented as previously described above

pulmonary luminescence in nude mice as that in M12-crtl cells at 24 and $48 \mathrm{~h}$ (Figures $7 \mathrm{a}$ and $\mathrm{b}$ ). This indicated that the miR-296-3p-ICAM-1 axis mediates the innate immune escaping of tumour cells in nude mice absent of immune response mediated by $T$ cells.

We reasoned that the explanation for the observed differential decreases in lung luminescent signals is most likely related to NK cell activity changes specifically to M12 that was like СTC in blood vessels. In view of the high activity of endogenous NK cell, we used the anti-asialo GM1 antibody, which can eliminate specifically NK cell activity in BALB/C nude mice ${ }^{25,26}$ to clarify NK cell function in destroying M12/CTC before their extravasation into lungs. As expected, NK cell depletion almost completely counteracted the differential levels of pulmonary retention in above all M12 variants at $24 \mathrm{~h}$ after intravenous inoculation 
(Figures 7c and d), indicating that evading immune surveillance of M12 mediated by the miR-296-3p-ICAM-1 axis is mainly dependent on NK cell in nude mice. Moreover, re-injection of ex vivo-expanded human NK cells into these nude mice significantly facilitated pulmonary clearance of M12-anti-miR-296-3p and -anti-miR-296-3p + ctrl cells in nude mice lungs at $48 \mathrm{~h}$ (Figures $7 \mathrm{c}$ and $\mathrm{d}$ ). These data suggest that the miR-296-3p-ICAM-1-NK axis enhances tumour cell resistance to NK cell, in turn probably promoting tumour cell extravasation and spread in vivo.

MiR-296-3p-ICAM-1-NK axis affects survival of M12 as CTC in peripheral blood. To verify the hypothesis that the miR-296-3p-ICAM-1-NK/CTC axis is involved in the process of malignant transition, we intravenously injected above M12 variants also stably expressing GFP into nude mice with or without pre-treatment of anti-asialo GM1 antibody 1 day before injection. Next to get evidence for the existence of circulating $\mathrm{M} 12$, we determined the quantity of GFP-positive/7-amino-actinomycin D (7-AAD)-negative cells in nude mouse peripheral blood by flow cytometry (Figure 8a; Supplementary Figures S9 and S10). Interestingly, circulating cells of M12 variants displayed similar number patterns (Figure $8 \mathrm{a}$, right panel) with the residual lung luminescence of living imaging (Figures $7 a$ and $b$ ). We found that anti-miR296-3p M12 showed reduced quantity of circulating cells at 24 and $36 \mathrm{~h}$ and this decrease was recovered by knockdowning ICAM-1 (Figure 8a, right panel).

Under conditions of endogenous NK cell depletion, the group of non-transferred human NK cells showed no differences in the number of circulating M12 later at $36 \mathrm{~h}$ (Figure $8 \mathrm{~b}$ ) whereas the group of re-transferred human NK cells showed different quantities of circulating cells among M12 variants (Figure $8 \mathrm{c}$ ). Not surprisingly, the quantity of miR-296-3p-silencing M12 in blood vessels remarkably decreased compared with counterparts control and anti-miR-296-3p+shICAM-1 M12 cells (Figure 8c). These results showed that the miR-296-3p-
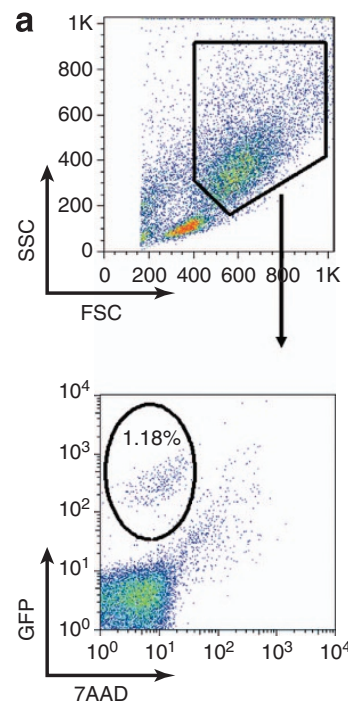

b

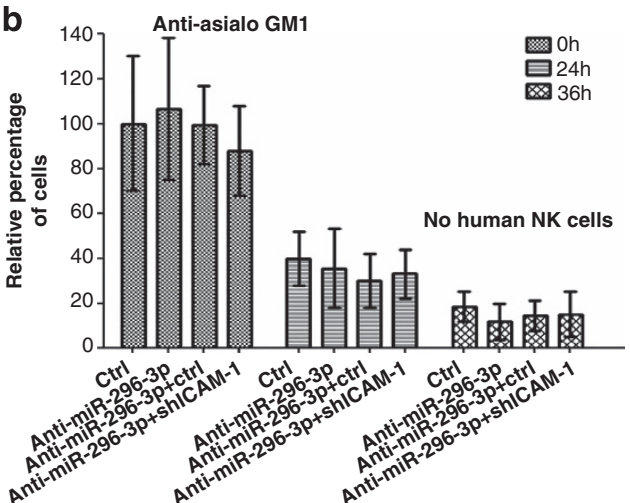

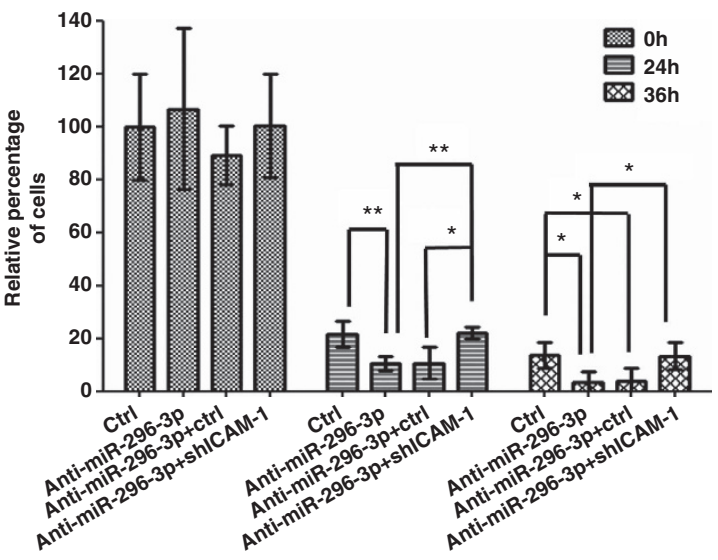

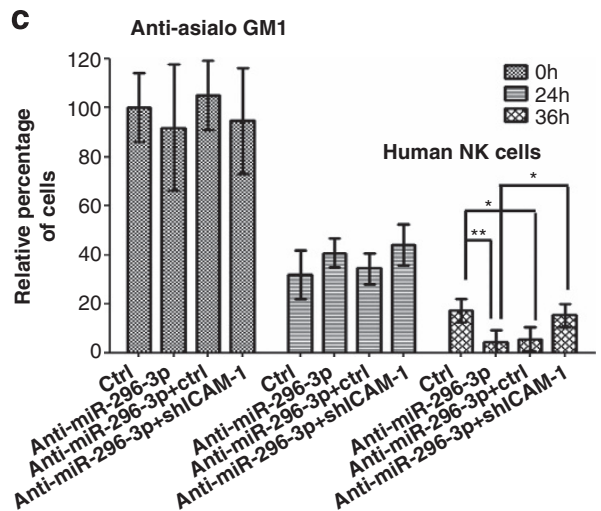

Figure 8 MiR-296-3p-ICAM-1-NK signalling axis affects survival of M12 in peripheral blood. (a) Quantitative analysis of the residual GFP-positive M12 in peripheral blood of nude mice treated with normal rabbit serum. The cells gated on FSC-SSC plot were analyzed (left upper panel) and the number in oval represented percentage of GFP ${ }^{+}$/ 7-AAD ${ }^{-}$viable M12 variant cells (left lower panel). The percentage of GFP ${ }^{+} \mathrm{M} 12$ variants at three time points of 0,24 and $36 \mathrm{~h}$ was normalized by being divided by percentage of GFP ${ }^{+}$cells in M12 control cells at 0 h. The data are representative of two independent experiments $(n=5)$ expressed as mean \pm S.E.M. (right graph). (b and c) Mice were intraperitoneally pre-treated anti-asialo GM1 antibody 1 day before intravenous inoculation of M12 variant cells. Quantitative analysis of residual GFP ${ }^{+}$M12 variants in peripheral blood of these mice transferred without (b) or with (c) human NK cells at $24 \mathrm{~h}$ after intravenous injection of tumour cells. The data were processed and presented as previously described 
ICAM-1-dependent NK- cell resistance is involved in survival and spread of M12 as CTC in blood vessels.

\section{Discussion}

The links between cancer cell evading immune surveillance and metastasizing to distant tissues are in heated discussion in recent years. Owing to the independence on specific tumour antigens, NK cell is considered as an antitumour innate immune effecter that can suppress cancer metastasis. ${ }^{27,28}$ The underlying mechanism of NK cell antitumour may lie in efficient elimination of $\mathrm{CTC}^{29,30}$ and preventing metastatic dissemination, although they are ineffective in primary solid tumours and distant metastases. To escape from NK cell lysis in vasculature, CTC may be coated with platelet-rich thrombi ${ }^{31}$ or downregulated the expression levels of IRF-1 (ref. 32) and NKG2D ligands. ${ }^{33}$ Except these mechanisms of CTC avoiding NK cell lysis, other mechanisms of tumour cell resistance against NK cells are requisite to be elucidated. In this study, we demonstrated a mechanism for survival of CTC with a property of reduced ICAM-1 expression, which may be acquired during EMT, in athymic nude mice characterized by the defective $T$ cell but high NK cell activity.

Screening of ligands by flow cytometry showed the expression levels of ICAM-1 and ULBP-2 in P69 were higher than those in M12 (Figure 3a), both of which are membrane proteins and involved in tumour cell lysis by NK cell. We found the lytic activity was significantly reduced after blockade of LFA-1/CD $11 \alpha$ on NK cells with the specific mAb (Figure 3e), which provided an evidence that ICAM-1 is involved in NK cell cytotoxicity to P69/M12. Of note, the mAb of LFA-1 did not completely eliminate the differential cytotoxicity between P69 and M12, and NKG2D signal pathway also greatly contributed to the NK cell susceptibility (Supplementary Figure S6). These data indicate that LFA-1/ICAM-1 partly mediates the differential NK cell susceptibility of P69/M12, and it probably could be co-stimulatory for the NKG2D-dependent NK cell cytotoxicity despite lack of direct evidence.

It has been reported that ICAM-1 is not sufficient to induce the degranulation of resting or IL-2 activated NK cells, ${ }^{34,35}$ and to enhance the degranulation of resting NK cells induced by NKG2D and 2B4 synergy. ${ }^{34}$ However, we observed the altered degranulation capacity of our ex vivo-expanded human NK cells with P69/M12 (Figure 2b). Furthermore, knockdown of ICAM-1 in P69 led to the decreased degranulation of NK cells, and conversely ectopic expression of ICAM-1 in M12 enhanced degranulation of NK cells (Figure 3d). Given that differential NK cell phenotypes and receptor expression patterns induced by various stimuli, ${ }^{19,36-38}$ we deduced that LFA-1/ICAM-1 can induce different outside-in signalling in ex vivo-expanded human NK cells stimulated by K562 aAPC feeder cells in this study.

MiRNAs is linked to cancer immunoediting from immunosurveillance to tumour escape in virus-infected ${ }^{10,39}$ and tumour cells ${ }^{40}$ by regulation of the expressions of some ligands such as MICA and MICB. However, whether/how miRNAs influence tumour cell evading from NK cell in vasculature remains unclear. In this study, we first observed negative correlation of the expressions of miR-296-3p and
ICAM-1 between P69 and M12 (Figures 4b-d and g) and in clinical PCa tissues (Figure 5), and showed that ectopic expression of miR-296-3p in P69 rendered highly resistant to NK cells whereas down-expression of miR-296-3p in M12 conferred sensitivity to NK cell (Figures 4a and 6). Recovery expression of ICAM-1 in P69 overexpressing miR-296-3p cells rescued the sensitivity to NK cell (Figure $6 b$ ), and knockdown of ICAM-1 in M12 silencing miR-296-3p cells suppressed the susceptibility to effectors (Figure 6c). Furthermore, blockade of LFA-1 on NK cells with specific mAbs counteracted the difference of sensitivity to NK cells between miR-296-3poverexpressing P69 and control cells or between miR-296-3pknockdowning M12 and control cells (Figure 6d), which strongly support the concept of miR-296-3p targeting ICAM-1.

Most importantly, the in vivo animal assays provided key evidences for the effect of miR-296-3p-ICAM-1 axis on M12/CTC escaping from NK cell lysis in blood vessels. We found that both pulmonary retention and survival in peripheral blood of M12-anti-miR-296-3p were less than those in M12-ctrl and M12-anti-miR-296-3p + shICAM-1 cells at $24 \mathrm{~h}$ and $36 / 48 \mathrm{~h}$ after injection into tail vein (Figures $7 \mathrm{a}$, b and $8 a)$. Interestingly, pulmonary retention and circulating survival of all M12 variants were comparable at $24 \mathrm{~h}$ under the depletion of mouse endogenous NK cells, but further re-injection of ex vivo-expanded human NK cells recovered the differential pulmonary retention and circulating survival (Figures 7c, $d$ and 8c). These data indicate that miR-296-3pICAM-1 axis increase CTC survival by acquiring resistance to NK cell lysis in circulation. During the early stage of EMT in the long-term transition process from P69 to M12 cells in nude mice ${ }^{15}$ cancer cells underwent evading immune surveillance by downregulation of ICAM-1, which contributes to decrease NK cell cytotoxicity. Our findings address that miRNA is a backdoor for malignantly transformed cell to escape from NK cell lysis and miR-296-3p has a role in PCa resistance to NK cell by targeting ICAM-1.

Finally, as summary in Figure 9, we demonstrate that a new miR-296-3p-ICAM-1 axis promotes $\mathrm{PCa}$ metastasis by possible enhancing survival of NK cell-resistant CTC. Our results also suggest that $\mathrm{PCa}$ highly expressing miR-296-3p may develop a novel strategy for evasion of NK cell immunity and has an increase in metastatic potential.

\section{Materials and Methods}

Reagents. The antibodies phycoerythrin (PE) anti-human CD16, PE antihuman LFA-1 (CD11 $\alpha$ ), PE anti-human NKG2D, PE anti-human NKP30, PE antihuman NKP44, PE anti-human NKP46, PE anti-human NKP80, PE anti-human DNAM-1 (CD226), PE anti-human MICA/B, PE anti-human LILRB1 (CD85j), PE anti-human CD48, PE anti-human CD112, allophycocyanin (APC) anti-human NCAM (CD56), APC anti-human ICAM-1 (CD54), APC anti-human CD244, APC anti-human HLA/B/C, fluorescein isothiocyanate (FITC) anti-human CD3, FITC anti-human KIR2DL1, FITC anti-human KIR2DL2/3, FITC anti-human KIR3DL1, PerCP/Cy5.5 anti-human LAMP-1 (CD107 $\alpha$, murine isotype controls IgG1k-PE, IgG2 $\alpha \mathrm{k}$, IgG2 $\beta \mathrm{k}$, IgG1k-FITC, IgG2 $\alpha \mathrm{k}-\mathrm{APC}, \lg \mathrm{G} 1, \lg \mathrm{G} 2 \beta \mathrm{k}$, secondary APC goat anti-mouse IgG, anti-human perforin and 7-AAD Viability Staining Solution were purchased from BioLegend, Inc., San Diego, CA, USA). Anti-human ULBP1, antihuman ULBP2, anti-human ULBP3, anti-human ULBP4, anti-human ULBP5 antibodies were obtained from Santa Cruz Biotechnology, Inc. (Dallas, TX, USA). Anti-human NKG2D mAb (clone 149810) for blockade assay and anti-human ICAM-1 polyclonal antibody for IHC staining were purchased from R\&D Systems, Inc. (Minneapolis, MN, USA). Anti-human CD11 $\alpha$ antibody for blockade assay was obtained from eBioscience, Inc. (San Diego, CA, USA). Calcein-AM was obtained 


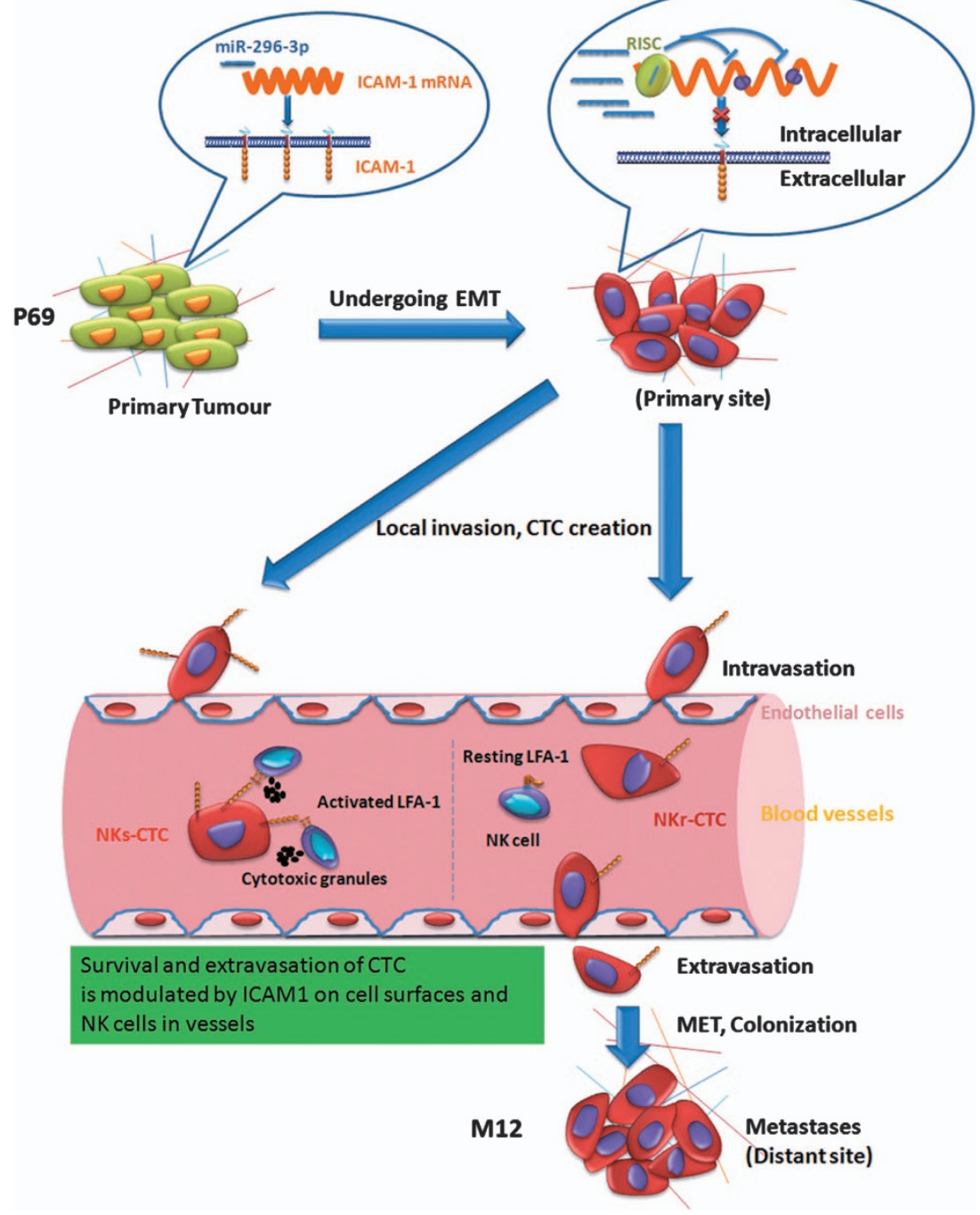

Figure 9 Schematic model of miR-296-3p-ICAM-1 axis promotes PCa metastasis by possible enhancing survival of NK cell-resistant CTC. During the early stage of EMT in the long-term malignant transition process from non-metastatic P69 to highly metastatic M12 in nude mice, ${ }^{15}$ some of tumour cells acquired genetic changes leading to highly expressing miR-296-3p that directly targeted ICAM-1 gene. These heterogenous tumour cells might invade locally and afterward intravasate into blood vessels to form CTC. Compared with NKs-CTC or parental P69 cells, NKr-CTC with low expression of ICAM-1 on cell surfaces inhibited by miR-296-3p were hardly destroyed by NK cells in blood vessels. The survived NKr-CTC might successively complete the remaining events of the invasion-metastasis cascade including extravasation, micrometastasis, MET and metastatic colonization. CTC, circulating tumour cell; EMT, epithelial-mesenchymal transition; NKs-CTC, natural killer cell-sensitive circulating tumour cell; NKr-CTC, natural killer cell-resistant circulating tumour cells; MET, mesenchymal-epithelial transition

from Sigma-Aldrich (St. Louis, MO, USA). The recombinant human IL-2 protein was purchased from PeproTech (Rocky Hill, NJ, USA).

Cell cultures. The establishment and characterization of the human prostate epithelial cell sublines P69 and M12 have been reported before. ${ }^{14,15,41}$ P69 and M12 were cultured in RPMI 1640 (Hyclone, Logan, UT, USA) supplemented with epidermal growth factor $(10 \mathrm{ng} / \mathrm{ml})$, dexamethasone $(0.1 \mu \mathrm{M})$, insulin, transferring, and selenious acid (ITS: insulin $5 \mu \mathrm{g} / \mathrm{ml}$, transferring $5 \mu \mathrm{g} / \mathrm{ml}$, and selenium $5 \mathrm{ng} / \mathrm{ml}$; Roche, Basel, Switzerland), and gentamicin $(0.05 \mathrm{mg} / \mathrm{ml})$. Artificial antigen presentation cells (aAPC) K562-CD19-CD64-CD86-CD137L-mlL21 were obtained from Dr. Dean Lee of the University of Texas MD Anderson Cancer Center with a material transfer agreement and were cultured in RPMl1640 medium supplemented with $10 \%$ of fetal bovine serum (FBS; Gibco, Grand Island, NY, USA), $1 \%$ of penicillin-streptomycin and $2 \mathrm{mM}$ L-Glutamine and $200 \mathrm{U} / \mathrm{ml} \mathrm{IL-2} \mathrm{(Peprotech,} \mathrm{USA).}$

Stable cell lines. Stable cell lines P69-ctrl and P69-miR-296-3p were generated by lentiviral infections with lentivirus vector $p G$ reenPuro vector (from System Biosciences, Mountain View, CA, USA, it can be used for GFP sorting and
Puromycin selection) or a construct expressing a synthetic double-stranded miR-296-3p oligonucleotide sequences, 5'-GATCCGGAGGGTTGGGTGGAGGC

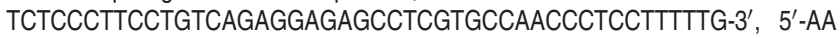
TTCAAAAAGGAGGGTTGGCACGAGGCTCTCCTCTGACAGGAAGGGAGAGCCT CCACCCAACCCTCCG-3', respectively.

Similarly, stable cell lines M12-ctrl and M12-anti-miR-296-3p were generated by lentiviral infections with lentivirus vector $p G$ reenPuro or a construct containing a synthetic double-stranded anti-miR-296-3p oligonucleotide sequences. 5'-GATCCGGAGGGTTGGCACGAGGCTCTCCCTTCCTGTCAGAGGAGAGCCT CCACCCAACCCTCCTTTTTG-3', 5'-AATTCAAAAAGGAGGGTTGGGTGGAGGCT CTCCTCTGACAGGAAGGGAGAGCCTCGTGCCAACCCTCCG-3'.

Real-time PCR quantification. Total RNAs were extracted from P69 and M12 using Trizol total RNA isolation reagent (Invitrogen, Carlsbad, CA, USA) following the manufacturer's instructions. The extracted total RNAs were reversely transcribed using miRNA-specific primers or random primers. ${ }^{42}$ Then $\mathrm{CDNAs}$ were used for PCR with the SYBR-Green Master PCR Mix (Applied Biosystems, Foster City, CA, USA) in triplicate. Data were normalized to an endogenous GAPDH or U6. 
Immunoblotting. Western blot analysis was performed using the following antibodies: anti-ICAM-1 (1:1000 dilution), anti-E-cadherin (\#3195, 1:1000 dilution; Cell Signaling Technology, Beverly, MA, USA) and anti- $\beta$-actin (A2228, 1:5000; Sigma-Aldrich).

NK cell purification and expansion in vitro. Human peripheral blood NK cells were acquired from healthy donors, and purified as described previously. ${ }^{19}$ Then, ex vivo expansion of human NK cells was carried out following the previous protocol described ${ }^{20}$ with minor modifications. K562 aAPCs were irradiated for 100 Gy via Gamma cell 3000 Elan (MDS Nordion, Ottawa, ON, Canada) and then mixed with PMBC at 2 to 1 (K562 aAPC: PBMC), and co-incubated in RPMI-1640 medium supplemented with $10 \%$ of $\mathrm{FBS}, 1 \%$ of penicillin-streptomycin, $2 \mathrm{mM}$ L-Glutamine and $50 \mathrm{U} / \mathrm{ml}$ of IL-2 (PeproTech, Rehovot, Israel) at $37{ }^{\circ} \mathrm{C}$ and $5 \% \mathrm{CO}_{2}$. In this study, only the expanded NK cells cultured from 14 to 21 days were used.

Flow cytometric analysis. In all, $1 \times 10^{6}$ of cells in single-cell suspension were stained by FITC, PE, PerCP-Cy5.5 or APC-conjugated antibodies at $4{ }^{\circ} \mathrm{C}$ for 30 min in PBS containing 1\% FBS. Samples were tested using BD FACSCalibur cytometer (BD Biosciences, San Jose, CA, USA) and data were analyzed via FlowJo software (Ashland, OR, USA).

Calcein-AM release assays for cytotoxicity. Calcein-AM release assays were performed as previously described ${ }^{43}$ with some modifications. In all, $1 \times 10^{6}$ of target cells were stained with $2 \mu \mathrm{g} / \mathrm{ml}$ calcein-AM (Sigma-Aldrich) for $30 \mathrm{~min}$ at $37^{\circ} \mathrm{C} .100 \mu \mathrm{l}$ of target cells and $100 \mu \mathrm{l}$ of NK cells were mixed at desired $\mathrm{E} / \mathrm{T}$ ratio and seeded in 96-well round bottom plates. The spontaneous or maximum calcein release of target cells was identified by spontaneous release well (only target cells in complete medium) and maximum release well (only target cells in $2 \%$ Triton $x-100$-containing medium), respectively. After incubation at $37^{\circ} \mathrm{C}$ and $5 \% \mathrm{CO} 2$ for $3 \mathrm{~h}, 100 \mu \mathrm{l}$ of each supernatant was harvested and transferred into a new plate for fluorescent detection. Samples were measured using microplate spectrofluorimeter (NOVOstar, BMG LABTECH, Offenburg, Germany) (excitation filter: 485, band-pass filter: 530). Specific lysis was calculated according to the formula [(test release-spontaneous release)/(maximum release-spontaneous release)] $\times 100$.

Degranulation assay. Degranulation assay was conducted as described previously with some modifications. ${ }^{19,44}$ In all, $1 \times 10^{5}$ of NK cells and $1 \times 10^{5}$ of target tumour cells were mixed in a total volume of $200 \mu \mathrm{l}$ and incubated at $37^{\circ} \mathrm{C}$ and $5 \% \mathrm{CO}_{2}$ for $2 \mathrm{~h}$ following addition of PerCP-Cy5.5-conjugated anti-human CD107 $\alpha$ (Biolegend, Inc.) with $20 \mu / \mathrm{ml}$. After washing and spinning down, $20 \mu / \mathrm{ml}$ of APC-conjugated anti-human CD56 Ab (Biolegend, Inc.) were added at to cells at $4^{\circ} \mathrm{C}$ for $30 \mathrm{~min}$.

Conjugation assay. Conjugation assay was conducted as described previously $^{22}$ with some modifications. The target cells were labelled with calcein-AM (Sigma-Aldrich) $3 \mu \mathrm{g} / \mathrm{ml}$ at a concentration of $1 \times 10^{6} \mathrm{cells} / \mathrm{ml}$ at $37^{\circ} \mathrm{C}$ for $30 \mathrm{~min}$. If target cells were transfected by lentivirus and expressed GFP, this step should be omitted. $1 \times 10^{5}$ of NK cells and $2 \times 10^{5}$ of the target cells were mixed, then briefly centrifugated at $20 \times g$ for 2 min and incubated at $37^{\circ} \mathrm{C}$. Cells were vortexed and then fixed using $1 \%$ paraformaldehyde when $0,10,30,60 \mathrm{~min}$ after incubation at $37^{\circ} \mathrm{C}$. Data were acquired by FACSArial (BD Biosciences). Conjugate pairs are represented by green and red double-positive events. Results are typically represented as the percentage of all NK cells that are in conjugates relative to the total number of NK cells.

Perforin polarization assay. The perforin polarization assay was conducted as previously described with some modifications. ${ }^{8}$ In all, $1 \times 10^{6}$ of cancer cells and $1 \times 10^{6}$ of NK cells were mixed and then incubated at $37^{\circ} \mathrm{C}$ for $20 \mathrm{~min}$. The mixture was transferred to poly-D-lysine-coated culture slides and incubated for $60 \mathrm{~min}$ at room temperature. The cells were fixed by $4 \%$ paraformaldehyde after washing and permeablized with $0.5 \%$ Triton X-100 in PBS. The cells were stained with anti-human perforin Ab (clone_G9) and with Alexa 568-conjugated secondary goat anti-mouse IgG Ab. Images of cells were captured using a confocal microscope (LSM710; CarlZeiss, Jena, Germany). Contacts between perforin-containing NK cell and target cell were identified, and the perforin was scored as polarized or not polarized by the visual appearance of the cell:cell conjugate.
IHC staining. Human prostate tissue array slides were stained with primary anti-ICAM-1 mAb at $4{ }^{\circ} \mathrm{C}$ overnight and then with secondary antibody conjugated with $\mathrm{HRP}$ at $37^{\circ} \mathrm{C}$ for $30 \mathrm{~min}$. The peroxidase-catalyzed product was visualized with the DAB Chromogen Kit (brown) followed by haematoxylin counterstain (blue). The threshold of ICAM-1-positive hue was determined by several control fields, which were optimal separation between brown and blue stained areas and data were presented by the ratio of ICAM-1-stained positive area/total area.

ISH histochemical staining. Human prostate tissue array slides were coincubated with Digoxigenin-conjugated oligonucleotide probes for human miR-296$3 p$ at $4{ }^{\circ} \mathrm{C}$ overnight and then stained with anti-DIG antibody conjugated with alkaline phosphatase at $37^{\circ} \mathrm{C}$ for $30 \mathrm{~min}$. The peroxidase-catalyzed product was visualized with the BCIP/NBT Chromogen Kit (blue) followed by nuclear fast red counterstain (red). The determination of threshold of miR-296-3p-positive hue and data presentation was similar to above-mentioned IHC method.

Immunofluorescence staining. Briefly, $1 \times 10^{5} / \mathrm{ml}$ of single cancer cell suspension was seeded to the poly-D-lysine-coated culture slide and incubated at $37^{\circ} \mathrm{C}, 5 \% \mathrm{CO}_{2}$ overnight. The cells were fixed by $4 \%$ paraformaldehyde, then stained with primary anti-human ICAM-1 mAb and subsequently with Alexa 568conjugated secondary goat anti-mouse IgG Ab followed by being counterstained with DAPI. Images of cells were captured using a confocal microscope (LSM510; Carl Zeiss)

Luciferase activity assay. The WT or mutated $3^{\prime}$-UTR of human ICAM-1 were amplified using PCR and cloned into the psiCHECK-2 vectors. The pshiCHECK-2 plasmid $(0.2 \mu \mathrm{g})$ was co-transfected into HEK 293T cells in 24-well plates with $0.4 \mu \mathrm{g}$ of control vector or plasmids expressing miR-296-3p (40 ng). Luciferase activity was measured at $24 \mathrm{~h}$ after transfection using the Dualluciferase reporter assay system (Promega, Madison, WI, USA) according to the manufacturer's instructions.

Migration and invasion assays by RTCA-DP. Cell migration and invasion assays were performed as described previously ${ }^{16,17}$ by using the xCELLigence RTCA-DP system (Roche, Mannheim, Germany). P69 and M12 were serum starved for $6 \mathrm{~h}$, then detached with $0.05 \%$ trypsin, cells were resuspended with serum-free medium and $100 \mu \mathrm{l}$ of suspension $\left(4 \times 10^{5} \mathrm{cell} / \mathrm{sl}\right)$ were added to the pre-equilibrated upper chamber of the CIM-plate. The migratory cells could be dynamically monitored using the RTCA-DP instrument. When used to determine the invasion assay, the CIM-plate is coated with Matrigel (BD Biosciences) $4 \mathrm{~h}$ before equilibration.

3D cultures. Cells were allowed to grow to $80 \%$ confluence of monolayer. $3 \mathrm{D}$ culture matrix was pre-thawed at $4^{\circ} \mathrm{C}$ overnight. Then added $10 \mu \mathrm{l}$ of $3 \mathrm{D}$ matrix into the inner well of $\mu$-slides and incubated at $37^{\circ} \mathrm{C}$ for at least $30 \mathrm{~min}$ until polymerization. Cells were trypsinized and resuspended at $1 \times 10^{5} \mathrm{cell} / \mathrm{s} / \mathrm{ml}$, then added $50 \mu$ l of cells onto the polymerized matrix. Confocal microscopy images were taken after 1-week growth. Immunofluorescene staining method was as described.

Luminescence imaging of cancer cells in animals. BALB/C nude/ nude mice at 8 weeks old were injected with $2.5 \times 10^{6}$ of ctrl, anti-miR-296-3p, anti-miR-296-3p + ctrl and anti-miR-296-3p + shICAM-1 M12/uc cells through the tail vein, respectively. Tumour sizes were evaluated at 0,24 and $48 \mathrm{~h}$ using the IVIS spectrum system (PerkinElmer Inc., Waltham, MA, USA) as previously described. ${ }^{45}$ For depletion of endogenous NK cells in mice, $50 \mu \mathrm{l}$ of anti-asialo GM1 (Cat. NO. 986-10001 from Wako Chemicals Gmbh, Neuss, Germany) or normal rabbit serum (Wako Chemicals Gmbh) was injected intraperitoneally 1 day before injection of tumour cells. To evaluate the function of ex vivo-expanded human NK cells, $2.5 \times 10^{7}$ of expanded human NK cells suspended in PBS or same volume of PBS were injected intravenously into nude mice at $24 \mathrm{~h}$ after tail vein injection of tumour cells. The pulmonary luminescence of $\mathrm{M} 12^{\text {luc }}$ cells in nude mice was determined by IVIS spectrum system at 0,24 and $48 \mathrm{~h}$, respectively. All animal studies were conducted with the approval and guidance of Shanghai JiaoTong University Medical Animal Ethics Committees.

Detection of circulating cancer cells in animals. In all, $100 \mu \mathrm{l}$ of blood sample per mouse was collected from the retro-orbital of an anaesthetic mouse at 0,24 and $36 \mathrm{~h}$ after intravenous inoculation of tumour cells, respectively. The blood sample was then stained with 7-AAD. The survival of M12 variants expressing GFP 
in peripheral blood of nude mice was determined by quantity of GFP-positive/7-AADnegative cells that were detected by BD FACSCalibur cytometer.

Statistical analysis. All data were presented as means \pm S.E.M. or means \pm S.D. Statistical calculations were performed with SPSS V18.0 software (IBM SPSS Inc., Chicago, IL, USA). Differences between individual groups were analyzed using the $t$-test (two-tailed and unpaired). A $P$-value of $<0.05\left(^{*}\right)$ or $\left.<0.01^{* \star}\right)$ or $<0.001{ }^{* * *}$ is considered significant. The correlation between the expression levels of miR-296-3p (ISH) and ICAM-1 (IHC) was determined by Spearman correlation test.

\section{Conflict of Interest}

The authors declare no conflict of interest.

Acknowledgements. This work was supported by grants National Key Program (973) for Basic Research of China (2012CB917101, 2011CB811303); National Natural Science Foundation (91129726, 31301066, 31071230); the PhD Programs Foundation of Ministry of Education of China (20120073110070); and Shanghai Committee of Science and Technology (11DZ2260200). We also acknowledge partial support from State Key Laboratory of Oncogenes and Related Genes, Shanghai Jiao Tong University School of Medicine.

1. Center MM, Jemal A, Lortet-Tieulent J, Ward E, Ferlay J, Brawley $O$ et al. Internationa variation in prostate cancer incidence and mortality rates. Eur Urol 2012; 61: 1079-1092.

2. Gupta GP, Massague J. Cancer metastasis: building a framework. Cell 2006; 127: 679-695.

3. Valastyan S, Weinberg RA. Tumor metastasis: molecular insights and evolving paradigms Cell 2011; 147: 275-292.

4. Schilling D, Todenhofer T, Hennenlotter J, Schwentner C, Fehm T, Stenzl A. Isolated, disseminated and circulating tumour cells in prostate cancer. Nat Rev Urol 2012; 9: 448-463.

5. Fidler IJ. The pathogenesis of cancer metastasis: the 'seed and soil' hypothesis revisited. Nat Rev Cancer 2003; 3: 453-458.

6. Jacob K, Sollier C, Jabado N. Circulating tumor cells: detection, molecular profiling and future prospects. Expert Rev Proteomics 2007; 4: 741-756.

7. Hanna N. Role of natural killer cells in control of cancer metastasis. Cancer Metastasis Rev 1982; 1: 45-64

8. Barber DF, Faure M, Long EO. LFA-1 contributes an early signal for NK cell cytotoxicity. J Immunol 2004; 173: 3653-3659

9. Becker JC, Dummer R, Hartmann AA, Burg G, Schmidt RE. Shedding of ICAM-1 from human melanoma cell lines induced by ifn-gamma and tumor necrosis factor-alpha. Functional consequences on cell-mediated cytotoxicity. J Immunol 1991; 147: 4398-4401.

10. Stern-Ginossar N, Gur C, Biton M, Horwitz E, Elboim M, Stanietsky N et al. Human microRNAs regulate stress-induced immune responses mediated by the receptor nkg2d. Nat Immunol 2008; 9: 1065-1073.

11. Himmelreich $\mathrm{H}$, Mathys A, Wodnar-Filipowicz A, Kalberer CP. Post-transcriptiona regulation of ULBP1 ligand for the activating immunoreceptor NKG2D involves $3^{3}$ untranslated region. Hum Immunol 2011; 72: 470-478.

12. Ueda R, Kohanbash G, Sasaki K, Fujita M, Zhu X, Kastenhuber ER et al. Dicer-regulated microRNAs 222 and 339 promote resistance of cancer cells to cytotoxic t-lymphocytes by down-regulation of ICAM-1. Proc Natl Acad Sci USA 2009; 106: 10746-10751.

13. Hu G, Gong AY, Liu J, Zhou R, Deng C, Chen XM. Mir-221 suppresses ICAM-1 translation and regulates interferon-gamma-induced ICAM-1 expression in human cholangiocytes. Am J Physiol Gastrointestinal Liver Physiol 2010; 298: G542-G550.

14. Bae VL, Jackson-Cook CK, Brothman AR, Maygarden SJ, Ware JL. Tumorigenicity of SV40 $T$ antigen immortalized human prostate epithelial cells: association with decreased epidermal growth factor receptor (EGFR) expression. Int J Cancer 1994; 58: 721-729.

15. Bae VL, Jackson-Cook CK, Maygarden SJ, Plymate SR, Chen J, Ware JL. Metastatic sublines of an SV40 large T antigen immortalized human prostate epithelial cell line. Prostate 1998; 34: 275-282

16. Ke N, Wang $\mathrm{X}, \mathrm{Xu} \mathrm{X}$, Abassi YA. The xcelligence system for real-time and label-free monitoring of cell viability. Methods $\mathrm{Mol}$ Biol 2011; 740: 33-43.

17. Golke A, Cymerys J, Slonska A, Dzieciatkowski T, Chmielewska A, Tucholska A et al. The xcelligence system for real-time and label-free analysis of neuronal and dermal cell response to equine herpesvirus type 1 infection. Pol J Vet Sci 2012; 15: 151-153.

18. Perl AK, Wilgenbus $P$, Dahl U, Semb H, Christofori G. A causal role for E-cadherin in the transition from adenoma to carcinoma. Nature 1998; 392: 190-193.

19. Liu X, Wang Y, Sun Q, Yan J, Huang J, Zhu S et al. Identification of microRNA transcriptome involved in human natural killer cell activation. Immunol Lett 2012; 143 208-217.
20. Denman CJ, Senyukov VV, Somanchi SS, Phatarpekar PV, Kopp LM, Johnson JL et al. Membrane-bound IL-21 promotes sustained ex vivo proliferation of human natural killer cells. PLoS One 2012; 7: e30264.

21. Waldhauer I, Steinle A. Proteolytic release of soluble UL16-binding protein 2 from tumor cells. Cancer Res 2006; 66: 2520-2526.

22. Gross CC, Brzostowski JA, Liu D, Long EO. Tethering of intercellular adhesion molecule on target cells is required for LFA-1-dependent NK cell adhesion and granule polarization. J Immunol 2010; 185: 2918-2926.

23. Betel D, Koppal A, Agius P, Sander C, Leslie C. Comprehensive modeling of microRNA targets predicts functional non-conserved and non-canonical sites. Genome Biol 2010; 11: R90.

24. Gupta GP, Nguyen DX, Chiang AC, Bos PD, Kim JY, Nadal C et al. Mediators of vascular remodelling co-opted for sequential steps in lung metastasis. Nature 2007; 446: 765-770.

25. Kasai M, Yoneda T, Habu S, Maruyama Y, Okumura K, Tokunaga T. In vivo effect of antiasialo gm1 antibody on natural killer activity. Nature 1981; 291: 334-335.

26. Martin-Fontecha A, Thomsen LL, Brett S, Gerard C, Lipp M, Lanzavecchia A et al. Induced recruitment of NK cells to lymph nodes provides IFN-gamma for $\mathrm{t}(\mathrm{h}) 1$ priming. Nat Immuno 2004; 5: 1260-1265.

27. Kim S, lizuka K, Aguila HL, Weissman IL, Yokoyama WM. In vivo natural killer cell activities revealed by natural killer cell-deficient mice. Proc Natl Acad Sci USA 2000; 97: 2731-2736.

28. Smyth MJ, Cretney E, Takeda K, Wiltrout RH, Sedger LM, Kayagaki N et al. Tumor necrosis factor-related apoptosis-inducing ligand (TRAIL) contributes to interferon gamma-dependent natural killer cell protection from tumor metastasis. J Exp Med 2001; 193: 661-670.

29. Hanna N, Burton RC. Definitive evidence that natural killer (NK) cells inhibit experimental tumor metastases in vivo. J Immunol 1981; 127: 1754-1758.

30. Barlozzari T, Reynolds CW, Herberman RB. In vivo role of natural killer cells: involvement of large granular lymphocytes in the clearance of tumor cells in anti-asialo gm1-treated rats. J Immunol 1983; 131: 1024-1027.

31. Gay LJ, Felding-Habermann B. Contribution of platelets to tumour metastasis. Nat Rev Cancer 2011; 11: 123-134.

32. Ksienzyk A, Neumann B, Nandakumar R, Finsterbusch K, Grashoff M, Zawatzky R et al. IRF-1 expression is essential for natural killer cells to suppress metastasis. Cancer Res 2011; 71: 6410-6418

33. Smyth MJ, Swann J, Kelly JM, Cretney E, Yokoyama WM, Diefenbach A et al. NKG2D recognition and perforin effector function mediate effective cytokine immunotherapy of cancer. J Exp Med 2004; 200: 1325-1335.

34. Bryceson YT, March ME, Barber DF, Ljunggren HG, Long EO. Cytolytic granule polarization and degranulation controlled by different receptors in resting NK cells. J Exp Med 2005; 202: 1001-1012.

35. Perez OD, Mitchell D, Jager GC, Nolan GP. LFA-1 signaling through $p 44 / 42$ is coupled to perforin degranulation in CD56 + CD8 + natural killer cells. Blood 2004; 104: 1083-1093.

36. Luhm J, Brand JM, Koritke P, Hoppner M, Kirchner H, Frohn C. Large-scale generation of natural killer lymphocytes for clinical application. J Hematotherapy Stem Cell Res 2002; 11 651-657.

37. Klingemann HG, Martinson J. Ex vivo expansion of natural killer cells for clinical applications. Cytotherapy 2004; 6: 15-22.

38. Ren H, Shen J, Tomiyama-Miyaji C, Watanabe M, Kainuma E, Inoue M et al. Augmentation of innate immunity by low-dose irradiation. Cell Immunol 2006; 244: 50-56.

39. Stern-Ginossar N, Elefant N, Zimmermann A, Wolf DG, Saleh N, Biton M et al. Host immune system gene targeting by a viral mirna. Science 2007; 317: 376-381.

40. Yadav D, Ngolab J, Lim RS, Krishnamurthy S, Bui JD. Cutting edge: down-regulation of $\mathrm{MHC}$ class I-related chain a on tumor cells by IFN-gamma-induced microRNA. J Immunol 2009; 182: 39-43.

41. Plymate SR, Tennant M, Birnbaum RS, Thrasher JB, Chatta G, Ware JL. The effect on the insulin-like growth factor system in human prostate epithelial cells of immortalization and transformation by simian virus-40 T antigen. J Clin Endocrinol Metab 1996; 81: 3709-3716.

42. Chen C, Ridzon DA, Broomer AJ, Zhou Z, Lee DH, Nguyen JT et al. Real-time quantification of microRNAs by stem-loop RT-PCR. Nucleic Acids Res 2005; 33: e179.

43. Neri S, Mariani E, Meneghetti A, Cattini L, Facchini A. Calcein-acetyoxymethyl cytotoxicity assay: standardization of a method allowing additional analyses on recovered effector cells and supernatants. Clin Diagnostic Lab Immunol 2001; 8: 1131-1135.

44. Alter G, Malenfant JM, Altfeld M. CD107A as a functional marker for the identification of natural killer cell activity. J Immunol Methods 2004; 294: 15-22.

45. Huang J, Yan J, Zhang J, Zhu S, Wang Y, Shi T et al. Sumo1 modification of pten regulates tumorigenesis by controlling its association with the plasma membrane. Nat Commun 2012; 3: 911.

(c) (i) (3) $\odot$ Cell Death and Disease is an open-access journal published by Nature Publishing Group. This work is licensed under a Creative Commons Attribution-NonCommercialNoDerivs 3.0 Unported License. To view a copy of this license, visit http://creativecommons.org/licenses/by-nc-nd/3.0/ 